\title{
ON PARAMETRICALLY QUASI-ELLIPTIC BOUNDARY PROBLEMS
}

\author{
VEIKKO T. PURMONEN
}

\section{Introduction}

Let $A(z, D)$ be a partial differential operator with a complex parameter $z$ such that the corresponding polynomial $A(z, \eta)$ is quasi-elliptic of type $\chi$, in which case $A(z, D)$ will be called parametrically quasi-elliptic of type $\chi$. Let $B_{1}(z, D), \ldots, B_{\varkappa}(z, D)$ be $\varkappa$ partial differential operators and set $B(z, D)=$ $\left(B_{1}(z, D), \ldots, B_{\varkappa}(z, D)\right)$. We consider the boundary value problem

$$
\left(A(z, D) u, \gamma_{0} B(z, D) u\right)=(f, g) \text {. }
$$

In the elliptic case this problem was investigated by M. S. Agranovič and M. I. Višik in [2]. In this paper the problem (P) is studied in the above setting with operators of more general types but, on the other hand, in the so-called canonical situation.

After the preliminary section we introduce in Section 2 the notion of a parametrically quasi-elliptic operator $A(z, D)$ and consider the equation $A(z, D) u=f$. Section 3 is devoted to the boundary problem $(\mathrm{P})$ with quasi-homogeneous operators. We prove there an a priori estimate which satisfies certain uniformity requirements, and study the unique solvability of $(\mathrm{P})$. The results are extended for the nonhomogeneous case in Section 4. As we shall finally (in 4.5) note, one obtains as a consequence a known result for boundary problems of the form

$$
\left(A(D) u+\lambda u, \gamma_{0} B(D) u\right)=(f, g)
$$

under mildly reduced assumptions. A brief remark concerning generalizations is also made.

In a forthcoming paper we shall study general initial-boundary value problems by making essential use of the results of this paper.

Acknowledgement. For financial support I am indebted to the Emil Aaltonen Foundation. 


\section{Preliminaries}

1.1. Let typical points in $\boldsymbol{R}^{n}$ and in its dual $\boldsymbol{R}^{n}=\boldsymbol{R}_{\eta}^{\boldsymbol{n}}$ be denoted by $y=\left(y^{\prime}, y_{n}\right)=\left(y_{1}, \ldots, y_{n-1}, y_{n}\right)$ and $\eta=\left(\eta^{\prime}, \eta_{n}\right)=\left(\eta_{1}, \ldots, \eta_{n-1}, \eta_{n}\right)$, respectively, and set

$$
\langle y, \eta\rangle=\left\langle y^{\prime}, \eta^{\prime}\right\rangle+y_{n} \eta_{n}=y_{1} \eta_{1}+\ldots+y_{n-1} \eta_{n-1}+y_{n} \eta_{n} .
$$

If $\alpha=\left(\alpha_{1}, \ldots, \alpha_{n}\right) \in N^{n}$ is a multi-index, an $n$-tuple of nonnegative integers $\alpha_{k} \in N$, we write

$$
D^{\alpha}=D_{y}^{\alpha}=D_{1}^{\alpha_{1}} \ldots D_{n}^{\alpha_{n}}
$$

where $D_{k}=-i \partial / \partial y_{k}$ with the imaginary unit $i \in C$. Likewise, we set

$$
\eta^{\alpha}=\eta_{1}^{\alpha_{1}} \ldots \eta_{n}^{\alpha_{n}}
$$

In what follows we shall use also the often more appropriate notations $y=(x, t)=$ $\left(x_{1}, \ldots, x_{n-1}, t\right)$ and $\eta=(\xi, \tau)=\left(\xi_{1}, \ldots, \xi_{n-1}, \tau\right)$.

The inverse $\mathscr{F}^{-1}$ of the Fourier transformation $\mathscr{F}=\mathscr{F}_{y}$,

$$
(\mathscr{F} u)(\eta)=\pi_{n} \int e^{-i\langle y, \eta\rangle} u(y) d y,
$$

is denoted by $\mathscr{F}_{\eta}$, and similarly $\mathscr{F}_{\xi}=\mathscr{F}_{x}{ }^{-1}$. Let $\mathscr{F}_{x}$ and $\mathscr{F}_{t}$ stand for the partial Fourier transformations, too,

where $\pi_{k}=(2 \pi)^{-k / 2}$.

$$
\begin{gathered}
\left(\mathscr{F}_{x} u\right)(\xi, t)=\pi_{n-1} \int e^{-i\langle x, \xi\rangle} u(x, t) d x, \\
\left(\mathscr{F}_{t} u\right)(x, \tau)=\pi_{1} \int e^{-i t \tau} u(x, t) d t,
\end{gathered}
$$

Note. It is convenient, sometimes, to let $H(w)$ stand for a function $H$ in variable $w$.

1.2. Let $m_{k}, k=0,1, \ldots, n$, be positive integers, $\mu=\max \left\{m_{k}\right\}, \quad q_{k}=\mu / m_{k}$, and set $q=\left(q^{\prime}, q_{n}\right)=\left(q_{1}, \ldots, q_{n-1}, q_{n}\right)$.

Consider a (complex-valued, appropriately defined) function $H=H(z, \eta)$, $z \in Z, \eta=(\xi, \zeta)$ with $\xi \in R^{n-1}, \zeta \in R$ or $\zeta \in C$, where the parameter set $Z$ is the sector $Z=Z\left(\omega_{1}, \omega_{2}\right) \subset C, \omega_{1} \leqq \omega_{2}$, defined by

$$
Z\left(\omega_{1}, \omega_{2}\right)=\left\{z \in C \mid \omega_{1} \leqq \arg z \leqq \omega_{2}\right\} .
$$

We shall say that $H$ is homogeneous with weight $\left(q_{0}, q\right)$ or $\left(q_{0}, q\right)$-homogeneous of degree $s \in \boldsymbol{R}$, and write $\left(q_{0}, q\right)$-deg $H=s$ if

for all $\lambda>0$, where

$$
H\left(\lambda^{q_{0}} z, \lambda^{q} \eta\right)=\lambda^{s} H(z, \eta)
$$

$$
\lambda^{q} \eta=\left(\lambda^{q_{1}} \eta_{1}, \ldots, \lambda^{q_{n}} \eta_{n}\right)
$$

the homogeneities with other weights are defined and indicated analogously. 
Next, we define

$$
\begin{aligned}
& \langle\xi\rangle=\left(\sum_{k=1}^{n-1}\left|\xi_{k}\right|^{m_{k}}\right)^{1 / \mu}, \quad \xi \in R^{n-1}, \\
& \langle\tau\rangle=|\tau|^{1 / q_{n},} \quad \tau \in \boldsymbol{R}, \\
& \langle\eta\rangle=\left(\langle\xi\rangle^{\mu}+\langle\tau\rangle^{\mu}\right)^{1 / \mu}, \quad \eta=(\xi, \tau) \in \boldsymbol{R}^{n}, \\
& \langle z\rangle=|z|^{1 / q_{0}}, \quad z \in C,
\end{aligned}
$$

and set furthermore, if $w=\xi$ or $\eta$,

$$
\begin{aligned}
& h(z, w)=\langle z\rangle+\langle w\rangle, \\
& K(z, w)=\left(1+\langle z\rangle^{2}+\langle w\rangle^{2}\right)^{1 / 2}, \\
& K(w)=K(0, w)=\left(1+\langle w\rangle^{2}\right)^{1 / 2} .
\end{aligned}
$$

Note that the $\langle\cdot\rangle$-functions and their possible sums are homogeneous of degree 1 with respect to the corresponding weights.

1.3. $H^{s}$-spaces. Let $\boldsymbol{R}_{+}^{n}=\left\{y=(x, t) \in \boldsymbol{R}^{n} \mid t>0\right\}$ and, when convenient, let $\Omega$ stand for $\boldsymbol{R}^{n}, \boldsymbol{R}_{+}^{n}$ or $\boldsymbol{R}^{n-1}$. The norms of the Lebesgue space $L^{2}(\Omega)$ and the (anisotropic) Sobolev space $H^{s}(\Omega)$ (see L. Hörmander [3], L. N. Slobodeckiĭ [7], L. R. Volevič-B. P. Panejah [9]), here employed for $s \geqq 0$, are then denoted by $\|\cdot\|_{\Omega}$ and $\|\cdot\|_{s, \Omega}$, respectively; $\Omega$ will be omitted in the case $\Omega=\boldsymbol{R}^{n}$, and replaced by the symbol + in the case $\Omega=\boldsymbol{R}_{+}^{n}$ and by $\gamma$ in the case $\Omega=\boldsymbol{R}^{\boldsymbol{n}-1}$. We recall the definitions of the $H^{s}(\Omega)$-spaces: Let $\mathscr{S}^{\prime}\left(\boldsymbol{R}^{n}\right)$ be the space of tempered distributions in $\boldsymbol{R}^{n}$. Then $H^{s}\left(\boldsymbol{R}^{n}\right)$ is defined by

$$
H^{s}\left(\boldsymbol{R}^{n}\right)=\left\{u \in \mathscr{S}^{\prime}\left(\boldsymbol{R}^{n}\right) \mid K(\eta)^{s} \mathscr{F} u \in L^{2}\left(\boldsymbol{R}^{n}\right)\right\}
$$

and the norm $\|\cdot\|_{s}$ by

$$
\|u\|_{s}=\left\|K(\eta)^{s} \mathscr{F} u\right\|
$$

The definition of $H^{s}\left(\boldsymbol{R}^{n-1}\right)$ is analogous, and

$$
\|u\|_{s, \gamma}=\left\|K(\xi)^{s} \mathscr{F}_{x} u\right\|_{\gamma} .
$$

The space $H^{s}\left(\boldsymbol{R}_{+}^{n}\right)$ consists of restrictions $R_{+} U$ of $U \in H^{s}\left(\boldsymbol{R}^{n}\right)$, and $\|\cdot\|_{s,+}$ is given by

$$
\|u\|_{s,+}=\inf \left\{\|U\|_{s} \mid U \in H^{s}\left(\boldsymbol{R}^{n}\right), R_{+} U=u\right\}
$$

here $R_{+}$is the operator restricting functions (distributions) defined on $\boldsymbol{R}^{n}$ to $\boldsymbol{R}_{+}^{n}$.

Clearly $H^{0}(\Omega)=L^{2}(\Omega)$, so that $\|\cdot\|_{0, \Omega}$ can be replaced by $\|\cdot\|_{\Omega}$.

It is well-known that $C_{0}^{\infty}\left(\boldsymbol{R}^{n}\right)$ is dense in $H^{s}\left(\boldsymbol{R}^{n}\right)$ and $C_{0}^{\infty}\left(\overline{\boldsymbol{R}}_{+}^{n}\right)$ in $H^{s}\left(\boldsymbol{R}_{+}^{n}\right)$; the symbol $C_{0}^{k}\left(\overline{\boldsymbol{R}}_{+}^{n}\right)$, for $k \in N$ or $k=\infty$, is used to denote the space of restrictions to $\overline{\boldsymbol{R}}_{+}^{n}=\left\{y=(x, t) \in \boldsymbol{R}^{n} \mid t \geqq 0\right\}$ of $C_{0}^{k}\left(\boldsymbol{R}^{n}\right)$-functions $\left(C^{k}\right.$-functions in $\boldsymbol{R}^{n}$ having compact support).

We recall also that $H^{s}(\Omega) \subset H^{r}(\Omega)$ algebraically and topologically for $s \geqq r$. 
1.4. Let $z \in \boldsymbol{C}$. The definition

$$
\|u\|_{z, s, \Omega}^{2}=\|u\|_{s, \Omega}^{2}+\langle z\rangle^{2 s}\|u\|_{\Omega}^{2}, \quad u \in H^{s}(\Omega)
$$

yields another norm on $H^{s}(\Omega)$, equivalent to $\|\cdot\|_{s, \Omega}$ for any fixed $z \in C$. Let $H_{z}^{s}(\Omega)=\left(H^{s}(\Omega),\|\cdot\|_{z, s, \Omega}\right)$ be the space $H^{s}(\Omega)$ with norm $\|\cdot\|_{z, s, \Omega}$.

Note that

and

$$
\begin{gathered}
\|u\|_{z, s}^{2}=\|u\|_{z, s, R^{n}}^{2} \sim \int K(z, \eta)^{2 s}|\mathscr{F} u|^{2} d \eta, \quad u \in H^{s}\left(\boldsymbol{R}^{n}\right), \\
\|u\|_{z, s, \gamma}^{2}=\|u\|_{z, s, R^{n-1}}^{2} \sim \int K(z, \xi)^{2 s}\left|\mathscr{F}_{x} u\right|^{2} d \xi, \quad u \in H^{s}\left(\boldsymbol{R}^{n-1}\right),
\end{gathered}
$$

$$
\|u\|_{z, s,+}^{2}=\|u\|_{z, s, R_{+}^{n}}^{2} \sim \inf \left\{\|U\|_{z, s} \mid U \in H^{s}\left(\boldsymbol{R}^{n}\right), R_{+} U=u\right\}, \quad u \in H^{s}\left(\boldsymbol{R}_{+}^{n}\right) ;
$$

here, as well as below, the notation $A \sim B$ for two expressions $A$ and $B$ means that

$$
C_{1} A \leqq B \leqq C_{2} A
$$

with two suitable positive constants $C_{1}$ and $C_{2}$ (only with admissible dependences).

Note. The symbol $C$ will be used, throughout this paper, to denote a generic positive constant.

1.5. Lemma. If $s \geqq r \geqq 0$, there is a constant $C>0$ such that, for all $z \in C$,

Proof. Since

$$
\|u\|_{z, r, \Omega} \leqq C\|u\|_{z, s, \Omega}, \quad u \in H^{s}(\Omega) .
$$

and

$$
\|u\|_{z, r, \Omega} \sim\|u\|_{r, \Omega}+\langle z\rangle^{r}\|u\|_{\Omega}
$$

we obtain

$$
\|u\|_{r, \Omega} \leqq C\|u\|_{s, \Omega},
$$

$$
\|u\|_{z, r, \Omega} \leqq C\left(\|u\|_{s, \Omega}+\left(1+\langle z\rangle^{s}\right)\|u\|_{\Omega}\right) \leqq C\|u\|_{z, s, \Omega} .
$$

1.6. If $X$ and $Y$ are two (complex) normed spaces, $\mathscr{L}(X ; Y)$ will denote the normed space of all bounded (linear) operators of $X$ into $Y$.

We recall that there exists an extension operator $E$ from $H^{s}\left(\boldsymbol{R}_{+}^{n}\right)$ into $H^{s}\left(\boldsymbol{R}^{n}\right)$, i.e., an operator $E \in \mathscr{L}\left(H^{s}\left(\boldsymbol{R}_{+}^{n}\right) ; H^{s}\left(\boldsymbol{R}^{n}\right)\right)$ satisfying

$$
R_{+} E u=u, \quad u \in H^{s}\left(R_{+}^{n}\right) .
$$

In fact, let $v$ be a positive integer and define for $u \in C_{0}^{\infty}\left(\overline{\boldsymbol{R}}_{+}^{n}\right)$

$$
\left(E_{v} u\right)(x, t)= \begin{cases}u(x, t) & \text { if } t \geqq 0 \\ \sum_{j=1}^{v+1} \lambda_{j} u(x,-j t) & \text { if } \quad t<0,\end{cases}
$$

where the coefficients $\lambda_{1}, \ldots, \lambda_{v+1}$ are determined by the system of linear equations

$$
\sum_{j=1}^{v+1}(-j)^{k} \lambda_{j}=1, \quad k=0, \ldots, v .
$$


Then for any $s \geqq 0$ with $s<v$ (note that then $C_{0}^{v}\left(\boldsymbol{R}^{n}\right) \subset H^{s}\left(\boldsymbol{R}^{n}\right)$ and $v \geqq$ $\left.\max \left\{k \in N \mid k \leqq s / q_{n}\right\}+1\right)$ the operator

extends to an operator

$$
E_{v}: C_{0}^{\infty}\left(\overline{\boldsymbol{R}}_{+}^{n}\right) \rightarrow C_{0}^{v}\left(\boldsymbol{R}^{n}\right)
$$

$$
E=E_{v} \in \mathscr{L}\left(H^{s}\left(\boldsymbol{R}_{+}^{n}\right) ; H^{s}\left(\boldsymbol{R}^{n}\right)\right)
$$

having the property (1) (see [7]).

Moreover, if $\beta=0, \ldots, v$ and we set for $u \in C_{0}^{\infty}\left(\overline{\boldsymbol{R}}_{+}^{n}\right)$

$$
\left(E_{v}^{(\beta)} u\right)(x, t)= \begin{cases}u(x, t) & \text { if } t \geqq 0 \\ \sum_{j=1}^{v+1}(-j)^{\beta} \lambda_{j} u(x,-j t) & \text { if } \quad t<0,\end{cases}
$$

then the operator $E^{(\beta)}=E_{v}^{(\beta)}$ mapping $C_{0}^{\infty}\left(\overline{\boldsymbol{R}}_{+}^{n}\right)$ into $C_{0}^{\nu-\beta}\left(\boldsymbol{R}^{n}\right)$ satisfies

$$
D_{t}^{\beta} E=E^{(\beta)} D_{t}^{\beta} .
$$

1.7. Lemma. Let $s \geqq 0$ and $r \geqq 0$ be given. Then there is a constant $C>0$ such that

for all $z \in \boldsymbol{C}$.

$$
\langle z\rangle^{r}\|u\|_{z, s, \Omega} \leqq C\|u\|_{z, s+r, \Omega}, \quad u \in H^{s+r}(\Omega)
$$

Proof. The statement follows from the inequality

$$
\langle z\rangle^{2 r}\|u\|_{s, \Omega}^{2} \leqq C\|u\|_{z, s+r, \Omega}^{2}, \quad u \in H^{s+r}(\Omega)
$$

whose proof in the case $\Omega=\boldsymbol{R}^{n}$ or $\boldsymbol{R}^{n-1}$ is straightforward, and which then in the case $\Omega=\boldsymbol{R}_{+}^{n}$ is obtained by use of the extension operator $E=E_{v}$ with $v>s+r$.

1.8. The trace operator $\gamma_{0}: C_{0}^{\infty}\left(\overline{\boldsymbol{R}}_{+}^{n}\right) \rightarrow C_{0}^{\infty}\left(\boldsymbol{R}^{n-1}\right)$ is defined by $\left(\gamma_{0} u\right)(x)=$ $u(x, 0)$ for $u \in C_{0}^{\infty}\left(\overline{\boldsymbol{R}}_{+}^{n}\right)$ and, for $s>q_{n} / 2$, extends by continuity to a continuous operator

(see, e.g., [8]).

$$
\gamma_{0}: u \mapsto \gamma_{0} u: H^{s}\left(\boldsymbol{R}_{+}^{n}\right) \rightarrow H^{s-q_{n} / 2}\left(\boldsymbol{R}^{n-1}\right)
$$

1.9. Lemma. If $s>q_{n} / 2$, there exists a constant $C>0$ such that

for all $z \in \boldsymbol{C}$.

$$
\left\|\gamma_{0} u\right\|_{z, s-q_{n} / 2, \gamma} \leqq C\|u\|_{z, s,+}, \quad u \in H^{s}\left(\boldsymbol{R}_{+}^{n}\right),
$$

Proof. Let $u \in C_{0}^{\infty}\left(\overline{\boldsymbol{R}}_{+}^{n}\right)$ and put $U=E u$, where $E=E_{v}$ with $v>s$. Then we have

$$
\left.\left\|\gamma_{0} u\right\|_{z, s-q_{n} / 2, \gamma}^{2} \sim \int K(z, \xi)^{2 s-q_{n} \mid\left(\mathscr{F}_{x}\right.} \gamma_{0} U\right)\left.(\xi)\right|^{2} d \xi,
$$


where

$$
\begin{aligned}
& \left|\left(\mathscr{F}_{x} \gamma_{0} U\right)(\xi)\right|^{2}=\left|\left(\mathscr{F}_{x} U\right)(\xi, 0)\right|^{2} \leqq \pi_{1}^{2}\left(\int|\mathscr{F} U| d \tau\right)^{2} \\
& \leqq \pi_{1}^{2} \int\left(K(z, \xi)^{2 q_{n}}+\tau^{2}\right)|\mathscr{F} U|^{2} d \tau \int \frac{d \tau}{K(z, \xi)^{2 q_{n}}+\tau^{2}} \\
& =\frac{1}{2} K(z, \xi)^{-q_{n}} \int\left(K(z, \xi)^{2 q_{n}}+\tau^{2}\right)|\mathscr{F} U|^{2} d \tau .
\end{aligned}
$$

Hence it follows that

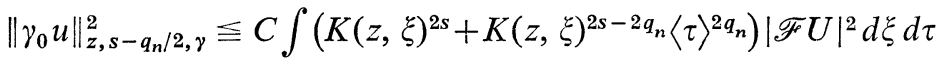

$$
\begin{aligned}
& \leqq C \int K(z, \eta)^{2 s}|\mathscr{F} U|^{2} d \eta \\
& \leqq C\|U\|_{z, s}^{2} \leqq C\|u\|_{z, s,+}^{2} \text {. }
\end{aligned}
$$

1.10. Lemma. Let $s \geqq 0$. There is a constant $C>0$ such that for any $\alpha \in N^{n}$ with $\langle\alpha, q\rangle \leqq s$ we have

for all $z \in \boldsymbol{C}$.

$$
\left\|D^{\alpha} u\right\|_{z, s-\langle\alpha, q\rangle,+} \leqq C\|u\|_{z, s,+}, \quad u \in H^{s}\left(\boldsymbol{R}_{+}^{n}\right),
$$

Proof. Suppose $u \in H^{s}\left(\boldsymbol{R}_{+}^{n}\right)$ and set $U=E u \in H^{s}\left(\boldsymbol{R}^{n}\right)$, where $E=E_{v}$ with $v>s$. If $r=\langle\alpha, q\rangle$, we have

$$
\left\|D^{\alpha} u\right\|_{z, s-\boldsymbol{r},+}^{2} \sim \int K(z, \eta)^{2 s-2 r}\left|\mathscr{F} D^{\alpha} U\right|^{2} d \eta=\int K(z, \eta)^{2 s-2 r} \eta^{2 \alpha}|\mathscr{F} U|^{2} d \eta .
$$

Now

so that

$$
\left|\eta^{\alpha}\right| \leqq C\langle\eta\rangle^{r}, \quad \eta \in \boldsymbol{R}^{n},
$$

$$
\left\|D^{\alpha} u\right\|_{z, s-r,+}^{2} \leqq C \int K(z, \eta)^{2 s}|\mathscr{F} U|^{2} d \eta \leqq C\|u\|_{z, s,+}^{2} .
$$

\section{Parametrically quasi-elliptic operators}

2.1. We shall consider partial differential operators of the form

$$
A(z, D)=\sum_{k q_{0}+\langle\alpha, q\rangle \leqq \mu} a_{k \alpha} z^{k} D^{\alpha} \quad\left(k \in N, \alpha \in N^{n}\right),
$$

where the coefficients $a_{k \alpha}$ are complex constants and the parameter $z \in \boldsymbol{C}$. The principal part $A^{0}(z, D)$ of $A(z, D)$ is given by

$$
A^{0}(z, D)=\sum_{k q_{0}+\langle\alpha, q\rangle=\mu} a_{k \alpha} z^{k} D^{\alpha},
$$

so that the corresponding polynomial

$$
A^{0}(z, \eta)=\sum_{k q_{0}+\langle\alpha, q\rangle=\mu} a_{k \alpha} z^{k} \eta^{\alpha},
$$

the principal symbol of $A(z, D)$, is $\left(q_{0}, q\right)$-homogeneous of degree $\mu$. 
2.2. Lemma. If $s \geqq \mu$, there is a constant $C>0$ such that

for all $z \in C$.

$$
\left\|A^{0}(z, D) u\right\|_{z, s-\mu} \leqq C\|u\|_{z, s}, \quad u \in H^{s}\left(\boldsymbol{R}^{n}\right),
$$

Proof. There exists a constant $C_{0}>0$ such that

$$
\left|A^{0}\left(z^{0}, \eta^{0}\right)\right| \leqq C_{0}, \quad z^{0} \in C, \eta^{0} \in R^{n}, h\left(z^{0}, \eta^{0}\right)=1 .
$$

For arbitrary $z \in \boldsymbol{C}$ and $\eta \in \boldsymbol{R}^{n}$ with $h(z, \eta)>0$ we find

$$
A^{0}(z, \eta)=h(z, \eta)^{\mu} A^{0}\left(z^{0}, \eta^{0}\right),
$$

where $z^{0}=h(z, \eta)^{-q_{0}} z, \eta^{0}=h(z, \eta)^{-q} \eta$ (see 1.2 and 2.1), and therefore

Thus we have

$$
\left|A^{0}(z, \eta)\right| \leqq C_{0} h(z, \eta)^{\mu}, \quad z \in \boldsymbol{C}, \eta \in \boldsymbol{R}^{n} .
$$

$$
\begin{aligned}
\left\|A^{0}(z, D) u\right\|_{z, s-\mu}^{2} & \sim \int K(z, \eta)^{2 s-2 \mu}\left|\mathscr{F} A^{0}(z, D) u\right|^{2} d \eta \\
& \leqq C \int K(z, \eta)^{2 s-2 \mu} h(z, \eta)^{2 \mu}|\mathscr{F} u|^{2} d \eta \\
& \leqq C\|u\|_{z, s}^{2} .
\end{aligned}
$$

2.3. Definition. The operator $A(z, D)$ is said to be parametrically quasielliptic if it satisfies the condition

$$
A^{0}(z, \eta) \neq 0, \quad z \in Z=Z\left(\omega_{1}, \omega_{2}\right), \eta \in R^{n}, h(z, \eta)>0,
$$

or, equivalently ( $c f .[3])$, if

$$
\left|A^{0}(z, \eta)\right| \geqq c_{0} h(z, \eta)^{\mu}, \quad z \in Z, \eta \in \boldsymbol{R}^{n},
$$

with some constant $c_{0}>0$.

Let us now assume that the condition (QE) is fulfilled. Consider $A^{0}(z, \xi, \tau)$ as polynomial in the complex variable $\tau$. Then there are functions $\tau_{k}=\tau_{k}(z, \xi)$, $k=1, \ldots, m_{n}$, continuous in $Z \times \boldsymbol{R}^{n-1}$, such that for each fixed $(z, \xi) \in Z \times \boldsymbol{R}^{n-1}$ they are the roots of the polynomial $A^{0}(z, \xi, \tau)$,

$$
A^{0}\left(z, \xi, \tau_{k}(z, \xi)\right)=0, \quad k=1, \ldots, m_{n} .
$$

2.4. Lemma. The roots $\tau_{k}(z, \xi), k=1, \ldots, m_{n}$, are $\left(q_{0}, q^{\prime}\right)$-homogeneous of degree $q_{n}$.

Indeed, we have

$$
A^{0}\left(z, \xi, \lambda-q_{n} \tau_{k}\left(\lambda^{q_{0}} z, \lambda^{q^{\prime}} \xi\right)\right)=0
$$

for every $\lambda>0$, and the function $\lambda \mapsto \lambda^{-q_{n}} \tau_{k}\left(\lambda^{q_{0}} z, \lambda^{q^{\prime}} \xi\right)$ from $\boldsymbol{R}_{+}$into $\boldsymbol{C}$ is continuous.

2.5. In what follows we shall mostly consider operators $A(z, D)$ which satisfy a somewhat stronger condition: 
Condition (QED). The operator $A(z, D)$ is parametrically quasi-elliptic of determined type $\chi, 1 \leqq \varkappa \leqq m_{n}$, that is, it satisfies $(Q E)$ and, moreover, the condition $(D)$ :

(D) For every $z \in Z$ and every $\xi \in \boldsymbol{R}^{n-1}$ with $h(z, \xi)>0$ the polynomial $A^{0}(z, \xi, \tau)$ in the complex variable $\tau$ has exactly $\varkappa$ roots $\tau_{j}^{+}(z, \xi), j=1, \ldots, \varkappa$, with positive imaginary part, $\operatorname{Im} \tau_{j}^{+}(z, \xi)>0$.

Remark. When $n \geqq 2$, (QE) implies (D) and hence (QED).

2.6. Theorem. Suppose that Condition $(Q E)$ is satisfied, and let $s \geqq \mu$. Given $\varrho>0$, there is a constant $C>0$ such that the a priori estimate

$$
\|u\|_{z, s} \leqq C\left\|A^{0}(z, D) u\right\|_{z, s-\mu}, \quad u \in H^{s}\left(\boldsymbol{R}^{n}\right),
$$

is valid for every $z \in Z$ with $\langle z\rangle \geqq \varrho$.

Furthermore, for every $z \in Z \backslash\{0\}$, the operator $A^{0}(z, D) \in \mathscr{L}\left(H_{z}^{s}\left(\boldsymbol{R}^{n}\right) ; H_{z}^{s-\mu}\left(\boldsymbol{R}^{n}\right)\right)$ is an isomorphism (for the locally convex structures). $\langle z\rangle>0$,

Hence

Proof. To prove (1), pick $u \in H^{s}\left(\boldsymbol{R}^{n}\right)$. By virtue of (QE) we then have, if

$$
|\mathscr{F} u| \leqq c_{0}^{-1} h(z, \eta)^{-\mu}\left|\mathscr{F} A^{0}(z, D) u\right| .
$$

$$
\|u\|_{z, s}^{2} \leqq C \int K(z, \eta)^{2 s} h(z, \eta)^{-2 \mu}\left|\mathscr{F} A^{0}(z, D) u\right|^{2} d \eta .
$$

Since here obviously

with $C=C(\varrho)$, we obtain

$$
h(z, \eta) \geqq C K(z, \eta)
$$

$$
\|u\|_{z, s}^{2} \leqq C \int K(z, \eta)^{2 s-2 \mu}\left|\mathscr{F} A^{0}(z, D) u\right|^{2} d \eta \leqq C\left\|A^{0}(z, D) u\right\|_{z, s-\mu}^{2} .
$$

It is clear that $A^{0}(z, D)$ is now an isomorphism of $H_{z}^{s}\left(\boldsymbol{R}^{n}\right)$ onto $H_{z}^{s-\mu}\left(\boldsymbol{R}^{n}\right)$ provided that it is surjective. This, however, is easy to see. Indeed, if $f \in H_{z}^{s-\mu}\left(\boldsymbol{R}^{n}\right)$, then

and

$$
u=\mathscr{F}_{\eta} A^{0}(z, \eta)^{-1} \mathscr{F} f \in H_{z}^{s}\left(\boldsymbol{R}^{n}\right)
$$

$$
A^{0}(z, D) u=\mathscr{F}_{\eta} A^{0}(z, \eta) \mathscr{F} u=f .
$$

\section{Boundary value problems}

\section{The case of principal parts}

3.1. Let $\varkappa$ be a positive integer, and let $B_{1}^{0}(z, D), \ldots, B_{\varkappa}^{0}(z, D)$ be $\varkappa$ operators defined by

$$
B_{j}^{0}(z, D)=\sum_{k q_{0}+\langle\alpha, q\rangle=u_{j}} b_{j k \alpha} z^{k} D^{\alpha}, \quad j=1, \ldots, x,
$$

where the coefficients $b_{j k \alpha}$ are complex constants, the parameter $z \in C$, and $\mu_{j} \geqq 0$. 
The corresponding polynomials

$$
B_{j}^{0}(z, \eta)=\sum_{k q_{0}+\langle\alpha, q\rangle=\mu_{j}} b_{j k \alpha} z^{k} \eta^{\alpha}, \quad j=1, \ldots, \varkappa,
$$

are then $\left(q_{0}, q\right)$-homogeneous, $\left(q_{0}, q\right)$-deg $B_{j}^{0}=\mu_{j}$.

Let $A^{0}(z, D)$ be the operator given by (1) of 2.1 and $P^{0}(z, D)$ the operator defined by

$$
P^{0}(z, D)=\left(A^{0}(z, D), \gamma_{0} B^{0}(z, D)\right)
$$

with $B^{0}(z, D)=\left(B_{1}^{0}(z, D), \ldots, B_{\varkappa}^{0}(z, D)\right)$.

For $s \in S$,

we set

$$
S=S\left(\mu, \mu_{j}\right)=\left\{s \in R \mid s \geqq \mu, s>\max \left\{\mu_{j}+q_{n} / 2, j=1, \ldots, x\right\}\right\},
$$

$$
\begin{gathered}
\mathscr{H}^{s}\left(\boldsymbol{R}^{n-1}\right)=\mathscr{H}^{s}\left(\boldsymbol{R}^{n-1} ; \mu_{j}\right)=\prod_{j=1}^{x} H^{s-\mu_{j}-q_{n} / 2}\left(\boldsymbol{R}^{n-1}\right), \\
\mathscr{H}^{s}\left(\boldsymbol{R}_{+}^{n}, \boldsymbol{R}^{n-1}\right)=\mathscr{H}^{s}\left(\boldsymbol{R}_{+}^{n}, \boldsymbol{R}^{n-1} ; \mu, \mu_{j}\right)=H^{s-\mu}\left(\boldsymbol{R}_{+}^{n}\right) \times \mathscr{H}^{s}\left(\boldsymbol{R}^{n-1}\right),
\end{gathered}
$$

and introduce similarly the spaces $\mathscr{H}_{z}^{s}\left(\boldsymbol{R}^{n-1}\right)$ and $\mathscr{H}_{z}^{s}\left(\boldsymbol{R}_{+}^{n}, \boldsymbol{R}^{n-1}\right)$; thus

and

$$
\mathscr{H}_{z}^{s}\left(\boldsymbol{R}^{n-1}\right)=\left(\mathscr{H}^{s}\left(\boldsymbol{R}^{n-1}\right),\||\cdot|\|_{z, s, \gamma}\right)
$$

with

$$
\mathscr{H}_{z}\left(\boldsymbol{R}_{+}^{n}, \boldsymbol{R}^{n-1}\right)=\left(\mathscr{H}^{s}\left(\boldsymbol{R}_{+}^{n}, \boldsymbol{R}^{n-1}\right),\||\cdot|\|_{z, s}\right)
$$

and

$$
\|\mid\|_{z, s, \gamma}^{2}=\sum_{j=1}^{\varkappa}\left\|g_{j}\right\|_{z, s-\mu_{j}-q_{n} / 2, \gamma}^{2}, \quad G=\left(g_{1}, \ldots, g_{\chi}\right) \in \mathscr{H}^{s}\left(\boldsymbol{R}^{n-1}\right)
$$

$$
\||| F\|_{z, s}^{2}=\|f\|_{z, s-\mu,+}^{2}+\||| G\|_{z, s, \gamma}^{2}, \quad F=(f, G) \in \mathscr{H}^{s}\left(\boldsymbol{R}_{+}^{n}, \boldsymbol{R}^{n-1}\right) .
$$

3.2. Lemma. If $s \in S$, there is a constant $C>0$ such that

for all $z \in C$.

$$
\|\| P^{0}(z, D) u\|\|_{z, s} \leqq C\|u\|_{z, s,+}, \quad u \in H^{s}\left(\boldsymbol{R}_{+}^{n}\right),
$$

Proof. Consider the right side of the inequality

$$
\|\| P^{0}(z, D) u \|_{z, s} \leqq C\left(\left\|A^{0}(z, D) u\right\|_{z, s-\mu,+}+\sum_{j=1}^{\varkappa}\left\|\gamma_{0} B_{j}^{0}(z, D) u\right\|_{z, s-\mu_{j}-q_{n} / 2, \gamma}\right) .
$$

By Lemmas 1.7 and 1.10,

$$
\begin{aligned}
\left\|A^{0}(z, D) u\right\|_{z, s-\mu,+} & \leqq \sum_{k q_{0}+\langle\alpha, q\rangle=\mu}\left|a_{k \alpha}\right||z|^{k}\left\|D^{\alpha} u\right\|_{z, s-\mu,+} \\
& \leqq C \sum_{k q_{0}+\langle\alpha, q\rangle=\mu}\langle z\rangle^{k q}\|u\|_{z, s-\mu+\langle\alpha, q\rangle,+} \\
& \leqq C\|u\|_{z, s,+}
\end{aligned}
$$

Making use of Lemma 1.9, we also obtain, as above,

$$
\left\|\gamma_{0} B_{j}^{0}(z, D) u\right\|_{z, s-\mu_{j}-q_{n} / 2, \gamma} \leqq C\left\|B_{j}^{0}(z, D) u\right\|_{z, s-\mu_{j},+} \leqq C\|u\|_{z, s,+} \cdot
$$


3.3. We now suppose that $A^{0}(z, D)$ satisfies Condition (QED).

3.3.1. Let $z \in Z$ and $\xi \in \boldsymbol{R}^{n-1}$ such that $h(z, \xi)>0$. We set

$$
A^{+}(z, \xi, \tau)=\prod_{j=1}^{\varkappa}\left(\tau-\tau_{j}^{+}(z, \xi)\right)=\sum_{k=0}^{\varkappa} \alpha_{k}^{+}(z, \xi) \tau^{\alpha-k},
$$

where the coefficients $\alpha_{k}^{+}(z, \xi)$ are $\left(q_{0}, q^{\prime}\right)$-homogeneous of degree $k q_{n}$, $k=0, \ldots, \varkappa$. Consequently, $A^{+}(z, \xi, \tau)$ is $\left(q_{0}, q\right)$-homogeneous,

$$
\left(q_{0}, q\right)-\operatorname{deg} A^{+}=x q_{n} .
$$

We shall also employ the polynomials (in $\tau$ )

$$
A_{v}^{+}(z, \xi, \tau)=\sum_{k=0}^{v} \alpha_{k}^{+}(z, \xi) \tau^{v-k}, \quad v=0, \ldots, \varkappa-1,
$$

which are $\left(q_{0}, q\right)$-homogeneous, too,

$$
\left(q_{0}, q\right)-\operatorname{deg} A_{v}^{+}=v q_{n} .
$$

The polynomials $A_{v}^{+}$are characterized by the following result, proved by a simple residue computation.

3.3.2. Lemma. If $z \in Z$ and $\xi \in \boldsymbol{R}^{n-1}$ with $h(z, \xi)>0$, then

$$
\frac{1}{2 \pi i} \int_{\Gamma} \frac{A_{\varkappa-v}^{+}(z, \xi, \zeta) \zeta^{k-1}}{A^{+}(z, \xi, \zeta)} d \zeta=\delta_{v k}, \quad v, k=1, \ldots, \varkappa,
$$

( $\delta_{v k}$ being the Kronecker symbol) for every rectifiable Jordan curve $\Gamma=\Gamma(z, \xi)$ which encircles the roots $\tau_{j}^{+}(z, \xi), j=1, \ldots, \varkappa$.

3.3.3. If $B_{j}^{0}(z, \eta)=B_{j}^{0}(z, \xi, \tau), 1 \leqq j \leqq \chi$, is the polynomial given by (1) of 3.1 , let $B_{j}^{\prime}(z, \xi, \tau)$ denote the uniquely determined polynomial in $\tau$ of degree less than $\chi$ such that

Then, since

$$
B_{j}^{0}(z, \xi, \tau) \equiv B_{j}^{\prime}(z, \xi, \tau) \bmod A^{+}(z, \xi, \tau) .
$$

$$
B_{j}^{0}(z, \xi, \tau) \equiv \lambda^{-\mu_{j}} B_{j}^{\prime}\left(\lambda^{q_{0}} z, \lambda^{q^{\prime}} \xi, \lambda^{q_{n}} \tau\right) \bmod A^{+}(z, \xi, \tau)
$$

for all $\lambda>0, B_{j}^{\prime}(z, \xi, \tau)$ is also $\left(q_{0}, q\right)$-homogeneous of degree $\mu_{j}$. Therefore, if we write

$$
B_{j}^{\prime}(z, \xi, \tau)=\sum_{k=1}^{\varkappa} \beta_{j k}(z, \xi) \tau^{k-1}, \quad j=1, \ldots, \varkappa,
$$

the coefficients $\beta_{j k}(z, \xi)$ are $\left(q_{0}, q^{\prime}\right)$-homogeneous,

$$
\left(q_{0}, q^{\prime}\right)-\operatorname{deg} \beta_{j k}=\mu_{j}-(k-1) q_{n} ;
$$

note that $\beta_{j k}=0$ when $(k-1) q_{n}>\mu_{j}$. 
3.3.4. The operators $A^{0}(z, D)$ and $B_{j}^{0}(z, D), j=1, \ldots, \varkappa$, are now connected by the following complementing (covering, Lopatinskiř-Šapiro) condition:

Condition (CC). For every $z \in Z$ and every $\xi \in \boldsymbol{R}^{n-1}$ with $h(z, \xi)>0$ the polynomials $B_{j}^{0}(z, \xi, \tau)$ in $\tau, j=1, \ldots, \chi$, are linearly independent modulo $A^{+}(z, \xi, \tau)$, or, what is the same,

$$
\operatorname{det} \mathscr{B}(z, \xi) \neq 0,
$$

where $\mathscr{B}(z, \xi)$ denotes the matrix $\left[\beta_{j k}(z, \xi)\right]$.

3.3.5. In order to employ Condition (CC) it is useful first to consider the ordinary differential equation

$$
A^{0}\left(z, \xi, D_{t}\right) \varphi=0
$$

with initial condition

$$
\gamma_{0} B^{0}\left(z, \xi, D_{t}\right) \varphi=c,
$$

where $c=\left(c_{1}, \ldots, c_{\varkappa}\right) \in \boldsymbol{C}^{\varkappa}$, and $h(z, \xi)>0$, of course. Let $\mathscr{S}\left(\boldsymbol{R}_{+}\right)$denote the space of functions $\varphi \in C^{\infty}\left(\overline{\boldsymbol{R}}_{+}\right)$such that $t^{\beta} D_{t}^{\alpha} \varphi(t) \rightarrow 0$ as $t \rightarrow \infty$ for all $\alpha, \beta \in N$.

3.3.6. Lemma. Assume Condition (QED) holds. Then Condition (CC) and the following two conditions are mutually equivalent:

(ES) Problem (4)-(5) admits a solution $\varphi \in \mathscr{S}\left(\boldsymbol{R}_{+}\right)$for all $c \in \boldsymbol{C}^{x}$.

(US) Problem (4)-(5) with $c=0$ admits only the trivial solution in $\mathscr{S}\left(\boldsymbol{R}_{+}\right)$.

It is convenient to recall briefly the proof of this lemma (cf. the proof of Proposition 4.2 of Lions-Magenes [4], p. 129), specially adapted for our purposes.

3.3.7. Proof of Lemma 3.3.6. Since the $\mathscr{S}\left(\boldsymbol{R}_{+}\right)$-solutions of (4) form a $x$ dimensional complex vector space $\mathscr{N}(z, \xi)$, the operator $T: \mathscr{N}(z, \xi) \rightarrow C^{\varkappa}$, defined by

$$
T \varphi=\gamma_{0} B^{0}\left(z, \xi, D_{t}\right) \varphi, \quad \varphi \in \mathscr{N}(z, \xi),
$$

is surjective if and only if it is injective, so that (ES) and (US) are equivalent.

Next we show that (CC) implies (ES). Under Condition (CC), the system of the equations

$$
\sum_{v=1}^{\varkappa} \beta_{j v}(z, \xi) g_{v}=c_{j}, \quad j=1, \ldots, \varkappa,
$$

has a unique solution $g(z, \xi)=\left(g_{1}(z, \xi), \ldots, g_{\varkappa}(z, \xi)\right)$ for every $c=\left(c_{1}, \ldots, c_{\varkappa}\right) \in C^{\varkappa}$; in fact

$$
g_{v}(z, \xi)=\sum_{k=1}^{\varkappa} \beta^{v k}(z, \xi) c_{k}, \quad v=1, \ldots, x,
$$

$\left[\beta^{\nu k}(z, \xi)\right]$ being the inverse matrix $\mathscr{B}^{-1}(z, \xi)$ of $\mathscr{B}(z, \xi)$. 
Let now $\Gamma$ be a rectifiable Jordan curve which surrounds the roots $\tau_{j}^{+}(z, \xi)$, $j=1, \ldots, x$. For any $t \geqq 0$, we define

$$
u(z, \zeta, t)=\frac{1}{2 \pi i} \int_{\Gamma} \sum_{\nu=1}^{\varkappa} g_{v}(z, \xi) \frac{A_{\varkappa-v}^{+}(z, \xi, \zeta)}{A^{+}(z, \xi, \zeta)} e^{i t \zeta} d \zeta .
$$

By (7), this becomes

$$
u(z, \xi, t)=\sum_{k=1}^{\varkappa} c_{k} N_{k}(z, \xi, t)
$$

where we have set

$$
N_{k}(z, \xi, t)=\frac{1}{2 \pi i} \int_{\Gamma} \frac{M_{k}(z, \xi, \zeta)}{A^{+}(z, \xi, \zeta)} e^{i t \zeta} d \zeta
$$

with

We have

$$
M_{k}(z, \zeta, \zeta)=\sum_{v=1}^{\varkappa} \beta^{v k}(z, \xi) A_{\varkappa-v}^{+}(z, \xi, \zeta) .
$$

$$
\begin{gathered}
N_{k}(z, \xi, \cdot) \in \mathscr{S}\left(\boldsymbol{R}_{+}\right), \\
A^{+}\left(z, \xi, D_{t}\right) N_{k}(z, \xi, t)=0,
\end{gathered}
$$

and furthermore, by Lemma 3.3.2,

$$
\begin{aligned}
& \gamma_{0} B_{j}^{0}\left(z, \xi, D_{t}\right) N_{k}(z, \xi, t)=\frac{1}{2 \pi i} \int_{\Gamma} \frac{M_{k}(z, \xi, \zeta)}{A^{+}(z, \xi, \zeta)} B_{j}^{\prime}(z, \xi, \zeta) d \zeta \\
& =\sum_{l=1}^{\varkappa} \beta_{j l}(z, \xi) \sum_{v=1}^{\varkappa} \beta^{v k}(z, \xi)\left(\frac{1}{2 \pi i} \int_{\Gamma} \frac{A_{\varkappa-v}^{+}(z, \xi, \zeta)}{A^{+}(z, \xi, \zeta)} \zeta^{l-1} d \zeta\right) \\
& =\sum_{l=1}^{\varkappa} \beta_{j l}(z, \xi) \beta^{l k}(z, \xi)=\delta_{j k} .
\end{aligned}
$$

Consequently, the function $u(z, \xi, \cdot)$ given by (9) is a solution of (4)-(5).

Finally, assume Condition (CC) does not hold. Then there are $z \in Z$ and $\xi \in \boldsymbol{R}^{n-1}$ with $h(z, \xi)>0$ such that (6) with $c_{j}=0$ has a nontrivial solution $g(z, \xi)$, that is, $g_{k}(z, \xi) \neq 0$ for some $k, 1 \leqq k \leqq \varkappa$. But then, for the function $u(z, \xi, \cdot) \in \mathscr{S}\left(\boldsymbol{R}_{+}\right)$ given by (8) we obtain

$$
\gamma_{0} D_{t}^{k-1} u(z, \xi, t)=\sum_{\nu=1}^{\varkappa} g_{v}(z, \xi)\left(\frac{1}{2 \pi i} \int_{\Gamma} \frac{A_{\varkappa-v}^{+}(z, \xi, \zeta)}{A^{+}(z, \xi, \zeta)} \zeta^{k-1} d \zeta\right)=g_{k}(z, \xi),
$$

which contradicts (US), and the proof is complete.

In connection with the preceding proof we have, moreover, the following two results required in the sequel.

3.3.8. Lemma. If Condition (CC) holds, then the functions $N_{1}, \ldots, N_{\varkappa}$, given by (10), form a basis of $\mathscr{N}(z, \xi)$. 
Proof. In fact, the functions in question are linearly independent, for if

$$
\sum_{k=1}^{\varkappa} c_{k} N_{k}(z, \xi, t)=0, \quad t \geqq 0,
$$

for some $c_{1}, \ldots, c_{\varkappa} \in C$, then it follows from (12) that

$$
0=\gamma_{0} B_{j}^{0}\left(z, \xi, D_{t}\right)\left(\sum_{k=1}^{\varkappa} c_{k} N_{k}(z, \xi, t)\right)=c_{j}, \quad j=1, \ldots, \varkappa .
$$

3.3.9. Lemma. If Condition (CC) holds, then the functions $M_{k}(z, \xi, \zeta)$, $k=1, \ldots, x$, given by $(11)$, are $\left(q_{0}, q\right)$-homogeneous,

$$
\left(q_{0}, q\right)-\operatorname{deg} M_{k}=(\varkappa-1) q_{n}-\mu_{k} .
$$

Proof. For all $\lambda>0$ we have

$$
\mathscr{B}(z, \xi) \mathscr{B}^{-1}(z, \xi)=\left[\delta_{j k}\right]=\mathscr{B}\left(\lambda^{q_{0}} z, \lambda^{q^{\prime}} \xi\right) \mathscr{B}^{-1}\left(\lambda^{q_{0}} z, \lambda^{q^{\prime}} \xi\right),
$$

so that, for $j, k=1, \ldots, \varkappa$,

$$
\sum_{v=1}^{\varkappa} \beta_{j v}(z, \xi) \beta^{v k}(z, \xi)=\delta_{j k}=\sum_{v=1}^{\varkappa} \beta_{j v}\left(\lambda^{q_{0}} z, \lambda^{q^{\prime}} \xi\right) \beta^{v k}\left(\lambda^{q_{0}} z, \lambda^{q^{\prime}} \xi\right) .
$$

If we use (3) and multiply the left equation by $\lambda^{\mu_{k}-\mu_{j}}$, we see that

$$
\sum_{\nu=1}^{\varkappa} \beta_{j v}(z, \xi) \beta^{v k}(z, \xi)=\delta_{j k}=\sum_{\nu=1}^{\varkappa} \beta_{j v}(z, \xi) \lambda^{\mu_{k}-(\nu-1) q_{n}} \beta^{\nu k}\left(\lambda q_{0} z, \lambda^{q^{\prime}} \xi\right) .
$$

Since $\mathscr{B}(z, \xi)$ is regular, it follows that

$$
\beta^{v k}(z, \xi)=\lambda^{\mu_{k}-(v-1) q_{n} \beta^{v k}}\left(\lambda q_{0} z, \lambda^{q^{\prime}} \xi\right)
$$

for $v, k=1, \ldots, x$; hence

$$
\left(q_{0}, q^{\prime}\right)-\operatorname{deg} \beta^{v k}=(v-1) q_{n}-\mu_{k} .
$$

From (2), (11) and (13) we now obtain, for all $i>0$,

$$
\begin{aligned}
& M_{k}\left(\lambda q_{0} z, \lambda^{q^{\prime}} \xi, \lambda q_{n} \zeta\right)=\sum_{\nu=1}^{\varkappa} \beta^{\nu k}\left(\lambda^{q_{0}} z, \lambda q^{\prime} \xi\right) A_{\varkappa-v}^{+}\left(\lambda q_{0} z, \lambda q^{\prime} \xi, \lambda q_{n} \zeta\right) \\
& =\sum_{\nu=1}^{\varkappa} \lambda^{(v-1) q_{n}-\mu_{k} \beta^{v k}}(z, \xi) \lambda^{(\varkappa-v) q_{n}} A_{\varkappa-v}^{+}(z, \xi, \zeta) \\
& =\lambda^{(\varkappa-1) q_{n}-\mu_{k}} M_{k}(z, \xi, \zeta) .
\end{aligned}
$$

3.4. Theorem. Suppose that Conditions (QED) and (CC) are satisfied, and that $s \in S$. Then, given any $\varrho>0$, there is a constant $C>0$ such that the a priori estimate

$$
\|u\|_{z, s,+} \leqq C\|\| P^{0}(z, D) u\|\|_{z, s}, \quad u \in H^{s}\left(\boldsymbol{R}_{+}^{n}\right),
$$

holds for all $z \in Z$ with $\langle z\rangle \geqq \varrho$. 
Furthermore, the operator $P^{0}(z, D) \in \mathscr{L}\left(H_{z}^{s}\left(\boldsymbol{R}_{+}^{n}\right) ; \mathscr{H}_{z}^{s}\left(\boldsymbol{R}_{+}^{n}, \boldsymbol{R}^{n-1}\right)\right)$ is an isomorphism for every $z \in Z \backslash\{0\}$.

The proof of Theorem 3.4 will be given in Sections 3.5 and 3.6.

3.5. Proof of the estimate $(A)$.

3.5.1. We begin by showing that the estimate $(\mathrm{A})$ is valid if and only if the estimate

$$
\|u\|_{z, s,+} \leqq C\|\| \gamma_{0} B^{0}(z, D) u\|\|_{z, s, \gamma}
$$

holds for every $u \in H^{s}\left(\boldsymbol{R}_{+}^{n}\right)$ with $A^{0}(z, D) u=0$.

That (A) implies (B) is obvious. To prove that (B) implies (A), let $u \in H^{s}\left(\boldsymbol{R}_{+}^{n}\right)$. Then

$$
f=A^{0}(z, D) u \in H^{s-\mu}\left(\boldsymbol{R}_{+}^{n}\right),
$$

and there exists $u_{1} \in H^{s}\left(\boldsymbol{R}_{+}^{n}\right)$ such that

$$
A^{0}(z, D) u_{1}=f
$$

and

$$
\left\|u_{1}\right\|_{z, s,+} \leqq C\|f\|_{z, s-\mu,+},
$$

where the constant $C>0$ does not depend on $f$. Indeed, if $E$ denotes the extension operator $E_{v}, v>s-\mu$, we set

$$
U_{1}=\mathscr{F}_{\eta} A^{0}(z, \eta)^{-1} \mathscr{F} E f .
$$

Then $U_{1} \in H^{s}\left(\boldsymbol{R}^{n}\right)$, and hence, by virtue of Theorem 2.6,

$$
\left\|U_{1}\right\|_{z, s} \leqq C\left\|A^{0}(z, D) U_{1}\right\|_{z, s-\mu}
$$

for all $z \in Z,\langle z\rangle \geqq \varrho$. Thus we have

and

$$
u_{1}=R_{+} U_{1} \in H^{s}\left(\boldsymbol{R}_{+}^{n}\right)
$$

$$
\left\|u_{1}\right\|_{z, s,+} \leqq C\|E f\|_{z, s-\mu} \leqq C\|f\|_{z, s-\mu,+} .
$$

Next, set $u_{0}=u-u_{1}$. Then $u_{0} \in H^{s}\left(\boldsymbol{R}_{+}^{n}\right)$ and, by $(1), A^{0}(z, D) u_{0}=0$. Consequently, (B) gives

$$
\left\|u_{0}\right\|_{z, s,+} \leqq C\left\|\gamma_{0} B^{0}(z, D) u_{0}\right\|_{z, s, \gamma} .
$$

In view of (2), (3), and Lemma 3.2, we thus obtain

$$
\begin{aligned}
\|u\|_{z, s,+} & \leqq\left\|u_{0}\right\|_{z, s,+}+\left\|u_{1}\right\|_{z, s,+} \\
& \leqq C\left(\|\| \gamma_{0} B^{0}(z, D) u\left\|_{z, s, \gamma}+\right\|\left\|\gamma_{0} B^{0}(z, D) u_{1}\right\| \|_{z, s, \gamma}\right)+\left\|u_{1}\right\|_{z, s,+} \\
& \leqq C\left(\|\| \gamma_{0} B^{0}(z, D) u\left\|_{z, s, \gamma}+\right\| u_{1} \|_{z, s,+}\right) \\
& \leqq C\left(\left\|A^{0}(z, D) u\right\|_{z, s-\mu,+}+\left\|\gamma_{0} B^{0}(z, D) u\right\|_{z, s, \gamma}\right) \\
& \leqq C\left\|\mid P^{0}(z, D) u\right\|_{z, s} .
\end{aligned}
$$


What we now have to prove is therefore the estimate (B); this will be done in the rest of this section.

3.5.2. If $u \in H^{s}\left(\boldsymbol{R}_{+}^{n}\right)$ and $A^{0}(z, D) u=0$, then

$$
0=\mathscr{F}_{x} A^{0}(z, D) u=A^{0}\left(z, \xi, D_{t}\right) \mathscr{F}_{x} u .
$$

Hence, for (almost) all $\xi \in \boldsymbol{R}^{n-1},\left(\mathscr{F}_{x} u\right)(\xi, \cdot)$ is an exponential solution of the equation (4) in 3.3 , so that it belongs to $\mathscr{S}\left(\boldsymbol{R}_{+}\right)$(cf. [1]) and, furthermore, to $\mathscr{N}(z, \xi)$. Accordingly, Lemma 3.3 .8 implies that

$$
\left(\mathscr{F}_{x} u\right)(\xi, t)=\sum_{k=1}^{x} c_{k}(z, \xi) N_{k}(z, \xi, t)
$$

where now (see the proof of Lemma 3.3.6)

$$
c_{k}(z, \xi)=\gamma_{0} B_{k}^{0}\left(z, \xi, D_{t}\right)\left(\mathscr{F}_{x} u\right)(\xi, t)=\gamma_{0} \mathscr{F}_{x} B_{k}^{0}(z, D) u .
$$

3.5.3. Choose an integer $v$ such that $v>s$ and $v>s / q_{n}+1 / 2$, and write again $E=E_{v}$.

We have

$$
\|u\|_{z, s,+}^{2} \leqq\|E u\|_{z, s}^{2} \sim \int K(z, \eta)^{2 s}|\mathscr{F} E u|^{2} d \eta .
$$

Here $\mathscr{F} E u=\mathscr{F}_{t} E \mathscr{F}_{x} u$, so that

$$
\mathscr{F} E u=\sum_{k=1}^{\varkappa} c_{k}(z, \xi)\left(\mathscr{F}_{t}\left(E N_{k}\right)(z, \xi, t)\right)(\tau) .
$$

Therefore it suffices to consider the integral

$$
\begin{aligned}
& \int K(z, \eta)^{2 s}\left|c_{k}(z, \xi)\left(\mathscr{F}_{t}\left(E N_{k}\right)(z, \xi, t)\right)(\tau)\right|^{2} d \eta \\
& \sim \int K(z, \xi)^{2 s}\left|c_{k}(z, \xi)\right|^{2}\left(\int\left|\left(\mathscr{F}_{t}\left(E N_{k}\right)(z, \xi, t)\right)(\tau)\right|^{2} d \tau\right) d \xi \\
& \quad+\int\left|c_{k}(z, \xi)\right|^{2}\left(\int\langle\tau\rangle^{2 s}\left|\left(\mathscr{F}_{t}\left(E N_{k}\right)(z, \xi, t)\right)(\tau)\right|^{2} d \tau\right) d \xi \\
& =I_{1}+I_{2} .
\end{aligned}
$$

3.5.4. Consider next the function

$$
N_{k}(z, \xi, t)=\frac{1}{2 \pi i} \int_{\Gamma_{\zeta}} \frac{M_{k}(z, \xi, \zeta)}{A^{+}(z, \xi, \zeta)} e^{i t \zeta} d \zeta,
$$

where $\Gamma_{\zeta}$ is a rectifiable Jordan curve encircling the roots $\tau_{j}^{+}(z, \xi), j=1, \ldots, \varkappa$.

There are $R>0$ and $\delta>0$ such that, for $j=1, \ldots, x$,

and

$$
\left|\tau_{j}^{+}\left(z^{0}, \xi^{0}\right)\right|<R
$$

$$
\left|\operatorname{Im} \tau_{j}^{+}\left(z^{0}, \xi^{0}\right)\right|>\delta
$$

for all $z^{0} \in Z$ and all $\xi^{0} \in \boldsymbol{R}^{n-1}$ with $h\left(z^{0}, \xi^{0}\right)=1$. Hence, with these $z^{0}$ and $\xi^{0}$, 
the curve $\Gamma_{\zeta}$ in (7) can always be deformed into the same Jordan curve, say, into $\Gamma_{R, \delta}$ consisting of the paths $|\zeta|=R, \operatorname{Im} \zeta \geqq \delta$ and $\operatorname{Im} \zeta=\delta,|\zeta| \leqq R$. From Lemma 2.4 it follows that for arbitrary $z \in Z$ and $\xi \in \boldsymbol{R}^{n-1}$ with $h(z, \xi)>0$ we can take $\Gamma_{\zeta}=h(z, \xi)^{q_{n}} \Gamma_{R, \delta}$.

The function $L_{k}$,

$$
L_{k}(z, \xi, \zeta)=\frac{M_{k}(z, \xi, \zeta)}{A^{+}(z, \xi, \zeta)}, \quad h(z, \xi)>0, \zeta \in C,
$$

is $\left(q_{0}, q\right)$-homogeneous of degree $-\mu_{k}-q_{n}$ (see (1) of 3.3 and Lemma 3.3.9), so that, in particular,

$$
L_{k}(z, \xi, \zeta)=h(z, \xi)^{-\mu_{k}-q_{n}} L_{k}\left(z^{0}, \xi^{0}, \zeta^{0}\right)
$$

when $(z, \xi, \zeta)=h(z, \xi)^{\left(q_{0}, q\right)}\left(z^{0}, \xi^{0}, \zeta^{0}\right)$. From this and the fact that, for a suitable constant $C>0$,

$$
\left|L_{k}\left(z^{0}, \xi^{0}, \zeta^{0}\right)\right| \leqq C
$$

for all $z^{0}, \xi^{0}$ with $h\left(z^{0}, \xi^{0}\right)=1$ and all $\zeta^{0} \in \Gamma_{R, \delta}$, we see that

$$
\left|L_{k}(z, \xi, \zeta)\right| \leqq C h(z, \xi)^{-\mu_{k}-q_{n}}
$$

for all $z, \xi$ with $h(z, \xi)>0$ and all $\zeta \in \Gamma_{\zeta}=h(z, \xi)^{q_{n}} \Gamma_{R, \delta}$.

By virtue of (7), (8) and (9) we thus obtain

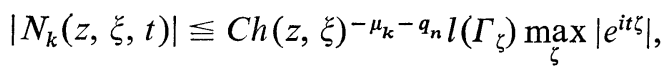

where $l\left(\Gamma_{\zeta}\right)$ is the length of $\Gamma_{\zeta}$. Since

and

$$
l\left(\Gamma_{\xi}\right)<(2+\pi) R h(z, \xi)^{q_{n}}
$$

we finally have

$$
\left|e^{i t \xi}\right| \leqq \exp \left(-h(z, \xi)^{q_{n}} \delta t\right),
$$

$$
\left|N_{k}(z, \xi, t)\right| \leqq C h(z, \xi)^{-\mu_{k}} \exp \left(-h(z, \xi)^{q_{n}} \delta t\right)
$$

for all $z$, $\xi$ with $h(z, \xi)>0$, and for all $t \geqq 0$.

3.5.5. We return now to (6). First, in the integral $I_{1}$ we have

$$
\begin{gathered}
\int\left|\left(\mathscr{F}_{t}\left(E N_{k}\right)(z, \xi, t)\right)(\tau)\right|^{2} d \tau=\int\left|\left(E N_{k}\right)(z, \xi, t)\right|^{2} d t \\
\leqq\left(1+(v+1) \sum_{j=1}^{v+1} \frac{1}{j}\left|\lambda_{j}\right|^{2}\right) \int_{0}^{\infty}\left|N_{k}(z, \xi, t)\right|^{2} d t
\end{gathered}
$$

(see 1.6), where, by (10),

$$
\int_{0}^{\infty}\left|N_{k}(z, \xi, t)\right|^{2} d t \leqq C h(z, \xi)^{-2 \mu_{k}-q_{n}} .
$$

Noting that $h(z, \xi) \sim K(z, \xi)$ when $\langle z\rangle \geqq \varrho$, we therefore obtain

$$
\begin{aligned}
I_{1} & \leqq C \int K(z, \xi)^{2 s}\left|\gamma_{0} \mathscr{F}_{x} B_{k}^{0}(z, D) u\right|^{2} K(z, \xi)^{-2 \mu_{k}-q_{n}} d \xi \\
& \leqq C\left\|\gamma_{0} B_{k}^{0}(z, D) u\right\|_{z, s-\mu_{k}-q_{n} / 2, \gamma}^{2} .
\end{aligned}
$$


3.5.6. To treat the integral $I_{2}$ let us rewrite it in the form

where

$$
I_{2}=\int\left|c_{k}(z, \xi)\right|^{2} H_{k}(z, \xi) d \xi,
$$

$$
H_{k}(z, \xi)=\int\langle\tau\rangle^{2 s}\left|\left(\mathscr{F}_{t}\left(E N_{k}\right)(z, \xi, t)\right)(\tau)\right|^{2} d \tau \text {. }
$$

3.5.7. First of all, we shall verify the continuity of $H_{k}(z, \xi)$ when $h(z, \xi)>0$.

To do this, let $\varepsilon>0$ be given, and let $z_{0} \in Z$ and $\xi_{0} \in R^{n-1}$ such that $h\left(z_{0}, \xi_{0}\right)>0$. Restrict the consideration to a neighbourhood of $\left(z_{0}, \xi_{0}\right)$, and notice that for all $(z, \xi)$ in this neighbourhood the curve $\Gamma_{\zeta}$ in (7) can be deformed into the same rectifiable Jordan curve, say, into $\Gamma$ with the property

$$
r=\min \{\operatorname{Im} \zeta \mid \zeta \in \Gamma\}>0
$$

(cf. 3.5.4). If $L_{k}$ denotes again the function given by (8), then there is $\delta_{\varepsilon}>0$ such that, for all $\zeta \in \Gamma$,

$$
\left|L_{k}(z, \xi, \zeta)-L_{k}\left(z_{0}, \xi_{0}, \zeta\right)\right|<\varepsilon
$$

whenever $\left|z-z_{0}\right|+\left|\xi-\xi_{0}\right|<\delta_{\varepsilon}$. In view of 1.6 , if $0 \leqq \beta \leqq v$, we thus have for $t \geqq 0$ (cf. 3.5.4)

$$
\begin{aligned}
& \left|\left(D_{t}^{\beta} N_{k}\right)(z, \xi, t)-\left(D_{t}^{\beta} N_{k}\right)\left(z_{0}, \xi_{0}, t\right)\right| \\
& \leqq \frac{1}{2 \pi} \int_{\Gamma}\left|L_{k}(z, \xi, \zeta)-L_{k}\left(z_{0}, \xi_{0}, \zeta\right)\right|\left|\zeta^{\beta}\right|\left|e^{i t \zeta}\right||d \zeta| \\
& \leqq C(\Gamma, v) \varepsilon e^{-r t}
\end{aligned}
$$

and then for $t<0$

$$
\begin{aligned}
& \left|\left(D_{t}^{\beta} E N_{k}\right)(z, \xi, t)-\left(D_{t}^{\beta} E N_{k}\right)\left(z_{0}, \xi_{0}, t\right)\right| \\
& =\left|\left(E^{(\beta)} D_{t}^{\beta} N_{k}\right)(z, \xi, t)-\left(E^{(\beta)} D_{t}^{\beta} N_{k}\right)\left(z_{0}, \xi_{0}, t\right)\right| \\
& \leqq \sum_{j=1}^{v+1}\left|(-j)^{\beta}\right|\left|\lambda_{j}\right|\left|\left(D_{t}^{\beta} N_{k}\right)(z, \xi,-j t)-\left(D_{t}^{\beta} N_{k}\right)\left(z_{0}, \xi_{0},-j t\right)\right| \\
& \leqq C(\Gamma, v) \varepsilon e^{-r|t|} ;
\end{aligned}
$$

hence, for all $t \in \boldsymbol{R}$,

$$
\left|\left(D_{t}^{\beta} E N_{k}\right)(z, \xi, t)-\left(D_{t}^{\beta} E N_{k}\right)\left(z_{0}, \xi_{0}, t\right)\right| \leqq C \varepsilon e^{-r|t|}
$$

if $\left|z-z_{0}\right|+\left|\xi-\xi_{0}\right|<\delta_{\varepsilon}$. This yields

$$
\begin{aligned}
& \left|\tau^{\beta}\left(\left(\mathscr{F}_{t}\left(E N_{k}\right)(z, \xi, t)\right)(\tau)-\left(\mathscr{F}_{t}\left(E N_{k}\right)\left(z_{0}, \xi_{0}, t\right)\right)(\tau)\right)\right| \\
& \leqq \pi_{1} \int\left|e^{-i t \tau}\left(\left(D_{t}^{\beta} E N_{k}\right)(z, \xi, t)-\left(D_{t}^{\beta} E N_{k}\right)\left(z_{0}, \xi_{0}, t\right)\right)\right| d t \\
& \leqq C \varepsilon,
\end{aligned}
$$

provided that $\left|z-z_{0}\right|+\left|\xi-\xi_{0}\right|<\delta_{\varepsilon}$. 
Next, a small computation shows that

$$
\begin{aligned}
& \left|H_{k}(z, \xi)-H_{k}\left(z_{0}, \xi_{0}\right)\right| \\
& =\left|\int\langle\tau\rangle^{2 s}\left(\left|\left(\mathscr{F}_{t}\left(E N_{k}\right)(z, \xi, t)\right)(\tau)\right|^{2}-\left|\left(\mathscr{F}_{t}\left(E N_{k}\right)\left(z_{0}, \xi_{0}, t\right)\right)(\tau)\right|^{2}\right) d \tau\right| \\
& \leqq 2 a b+b^{2},
\end{aligned}
$$

where

$$
\begin{aligned}
& a=\left(\int\langle\tau\rangle^{2 s}\left|\left(\mathscr{F}_{t}\left(E N_{k}\right)\left(z_{0}, \xi_{0}, t\right)\right)(\tau)\right|^{2} d \tau\right)^{1 / 2}, \\
& b=\left(\int\langle\tau\rangle^{2 s}\left|\left(\mathscr{F}_{t}\left(E N_{k}\right)(z, \xi, t)\right)(\tau)-\left(\mathscr{F}_{t}\left(E N_{k}\right)\left(z_{0}, \xi_{0}, t\right)\right)(\tau)\right|^{2} d \tau\right)^{1 / 2} .
\end{aligned}
$$

With $0 \leqq \beta \leqq \nu$ we have (see 1.6)

$$
\begin{aligned}
& \left|\tau^{\beta}\left(\mathscr{F}_{t}\left(E N_{k}\right)\left(z_{0}, \xi_{0}, t\right)\right)(\tau)\right|=\left|\left(\mathscr{F}_{t}\left(E^{(\beta)} D_{t}^{\beta} N_{k}\right)\left(z_{0}, \xi_{0}, t\right)\right)(\tau)\right| \\
& \quad=\pi_{1}\left|\int_{0}^{\infty} e^{-i t \tau}\left(D_{t}^{\beta} N_{k}\right)\left(z_{0}, \xi_{0}, t\right) d t+\sum_{j=1}^{\nu+1}(-j)^{\beta} \lambda_{j} \int_{-\infty}^{0} e^{-i t \tau}\left(D_{t}^{\beta} N_{k}\right)\left(z_{0}, \xi_{0},-j t\right) d t\right|,
\end{aligned}
$$

and since it follows from (9) that

$$
\left|\left(D_{t}^{\beta} N_{k}\right)\left(z_{0}, \xi_{0}, t\right)\right| \leqq C h\left(z_{0}, \xi_{0}\right)^{-\mu_{k}-q_{n}} e^{-r t},
$$

we obtain (cf. 3.5.4)

Hence we get

$$
\left|\tau^{\beta}\left(\mathscr{F}_{t}\left(E N_{k}\right)\left(z_{0}, \xi_{0}, t\right)\right)(\tau)\right| \leqq C h\left(z_{0}, \xi_{0}\right)^{-\mu_{k}-q_{n}} .
$$

$$
\begin{aligned}
a^{2} & \leqq C \int \frac{\langle\tau\rangle^{2 s}}{\left(1+\tau^{2}\right)^{v}}\left|\left(1+|\tau|^{v}\right)\left(\mathscr{F}_{t}\left(E N_{k}\right)\left(z_{0}, \xi_{0}, t\right)\right)(\tau)\right|^{2} d \tau \\
& \leqq C\left(z_{0}, \xi_{0}\right) \int \frac{|\tau|^{2 s / q_{n}}}{\left(1+\tau^{2}\right)^{v}} d \tau \\
& \leqq C\left(z_{0}, \xi_{0}\right),
\end{aligned}
$$

since $v>s / q_{n}+1 / 2$. From (12) we derive in the same way that

$$
b^{2} \leqq C \varepsilon .
$$

Therefore, it finally follows from (13) that

$$
\left|H_{k}(z, \xi)-H_{k}\left(z_{0}, \xi_{0}\right)\right| \leqq C \varepsilon
$$

whenever $\left|z-z_{0}\right|+\left|\xi-\xi_{0}\right|<\delta_{\varepsilon}$.

3.5.8. There exists a constant $C>0$ such that

$$
H_{k}\left(z^{0}, \xi^{0}\right) \leqq C, \quad z^{0} \in Z, \quad \xi^{0} \in R^{n-1}, h\left(z^{0}, \xi^{0}\right)=1 .
$$

Let then $z \in Z$ and $\xi \in \boldsymbol{R}^{n-1}$ with $h(z, \xi)>0$, let $\lambda>0$, and consider

$$
H_{k}\left(\lambda^{q_{0}} z, \lambda^{q^{\prime}} \xi\right)=\int\langle\tau\rangle^{2 s}\left|\left(\mathscr{F}_{t}\left(E N_{k}\right)\left(\lambda^{q_{0}} z, \lambda^{q^{\prime}} \xi, t\right)\right)(\tau)\right|^{2} d \tau .
$$


In the case $t \geqq 0$ we obtain (see 3.5 .4 )

so that, for all $t \in \boldsymbol{R}$,

$$
N_{k}\left(\lambda^{q_{0}} z, \lambda^{q^{\prime}} \xi, t\right)=\lambda^{-\mu_{k}} N_{k}\left(z, \xi, \lambda^{q_{n}} t\right),
$$

Consequently,

$$
\left(E N_{k}\right)\left(\lambda^{q_{0}} z, \lambda^{q^{\prime}} \xi, t\right)=\lambda^{-\mu_{k}}\left(E N_{k}\right)\left(z, \xi, \lambda^{q_{n}} t\right) .
$$

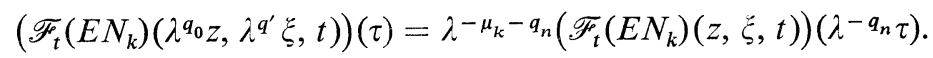

Hence we see that

$$
H_{k}\left(\lambda^{q_{0}} z, \lambda^{q^{\prime}} \xi\right)=\lambda^{-2 \mu_{k}-2 q_{n}} \int\langle\tau\rangle^{2 s}\left|\left(\mathscr{F}_{t}\left(E N_{k}\right)(z, \xi, t)\right)\left(\lambda^{-q_{n}} \tau\right)\right|^{2} d \tau=\lambda^{2 s-2 \mu_{k}-q_{n}} H_{k}(z, \xi),
$$

from which it follows, by (14), that

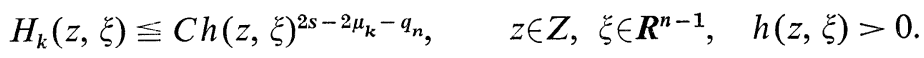

Now we return to the integral $I_{2}$ and reach the conclusion that

whence

$$
\begin{aligned}
& I_{2}=\int\left|c_{k}(z, \xi)\right|^{2} H_{k}(z, \xi) d \xi \leqq C \int h(z, \xi)^{2 s-2 \mu_{k}-q_{n}}\left|c_{k}(z, \xi)\right|^{2} d \xi
\end{aligned}
$$

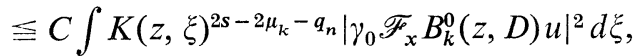

$$
I_{2} \leqq C\left\|\gamma_{0} B_{k}^{0}(z, D) u\right\|_{z, s-\mu_{k}-q_{n} / 2, \gamma}^{2} .
$$

3.5.9. By combining (4), (5), (6), (11), and (15) we finally obtain

$$
\begin{aligned}
\|u\|_{z, s,+}^{2} & \leqq C \sum_{k=1}^{\varkappa} \int K(z, \xi)^{2 s}\left|c_{k}(z, \xi)\left(\mathscr{F}_{t}\left(E N_{k}\right)(z, \xi, t)\right)(\tau)\right|^{2} d \eta \\
& \leqq C \sum_{k=1}^{\varkappa}\left\|\gamma_{0} B_{k}^{0}(z, D) u\right\|_{z, s-\mu_{k}-q_{n} / 2, \gamma}^{2} .
\end{aligned}
$$

This completes the proof of the estimate (B).

3.6. Proof of the latter part of Theorem 3.4. To show that $P^{0}(z, D)$ is an isomorphism of $H_{z}^{s}\left(\boldsymbol{R}_{+}^{n}\right)$ onto $\mathscr{H}_{z}^{s}\left(\boldsymbol{R}_{+}^{n}, \boldsymbol{R}^{n-1}\right)$, for every $z \in Z \backslash\{0\}$, we shall, in fact, construct its inverse operator. In what follows, let $E=E_{v}, v>s$.

3.6.1. Fix $z \in Z \backslash\{0\}$ and define

$$
K_{0} f=R_{+} \mathscr{F}_{\eta} A^{0}(z, \eta)^{-1} \mathscr{F} E f, \quad f \in H_{z}^{s-\mu}\left(\boldsymbol{R}_{+}^{n}\right) .
$$

Then $K_{0}$ is an operator mapping $H_{z}^{s-\mu}\left(\boldsymbol{R}_{+}^{n}\right)$ into $H_{z}^{s}\left(\boldsymbol{R}_{+}^{n}\right)$. Indeed, $E f \in H_{z}^{s-\mu}\left(\boldsymbol{R}^{n}\right)$ and

so that

$$
\begin{aligned}
\left\|\mathscr{F}_{\eta} A^{0}(z, \eta)^{-1} \mathscr{F} E f\right\|_{z, s}^{2} & \leqq C \int K(z, \eta)^{2 s}\left|A^{0}(z, \eta)^{-1} \mathscr{F} E f\right|^{2} d \eta \\
& \leqq C \int K(z, \eta)^{2 s} h(z, \eta)^{-2 \mu}|\mathscr{F} E f|^{2} d \eta \\
& \leqq C\|E f\|_{z, s-\mu}^{2},
\end{aligned}
$$

$$
\left\|K_{0} f\right\|_{z, s,+}^{2} \leqq C\|E f\|_{z, s-\mu}^{2} \leqq C\|f\|_{z, s-\mu,+}^{2} ;
$$


this implies, furthermore, that

$$
K_{0} \in \mathscr{L}\left(H_{z}^{s-\mu}\left(\boldsymbol{R}_{+}^{n}\right) ; H_{z}^{s}\left(\boldsymbol{R}_{+}^{n}\right)\right) .
$$

3.6.2. For $j=1, \ldots, x$ we set

$$
K_{j} g=\mathscr{F}_{\xi}\left(N_{j} \mathscr{F}_{x} g\right), \quad g \in H_{z}^{s-\mu_{j}-q_{n} / 2}\left(\boldsymbol{R}^{n-1}\right) .
$$

First, one easily checks (see 3.5.5) that $K_{j} g \in L^{2}\left(\boldsymbol{R}_{+}^{n}\right)$, at least. Since now

and so

$$
E K_{j} g=\mathscr{F}_{\xi}\left(\left(E N_{j}\right) \mathscr{F}_{x} g\right)
$$

we obtain

$$
\mathscr{F} E K_{j} g=\left(\mathscr{F}_{t} E N_{j}\right) \mathscr{F}_{x} g,
$$

$$
\begin{aligned}
& \int K(z, \eta)^{2 s}\left|\mathscr{F} E K_{j} g\right|^{2} d \eta \\
& \sim \int K(z, \xi)^{2 s}\left(\int\left|\mathscr{F}_{t} E N_{j}\right|^{2} d \tau\right)\left|\mathscr{F}_{x} g\right|^{2} d \xi+\int\left(\int\langle\tau\rangle^{2 s}\left|\mathscr{F}_{t} E N_{j}\right|^{2} d \tau\right)\left|\mathscr{F}_{x} g\right|^{2} d \xi \\
& =I_{1}+I_{2} .
\end{aligned}
$$

Here we have (see 3.5.5)

$$
I_{1} \leqq C \int K(z, \xi)^{2 s} h(z, \xi)^{-2 \mu_{j}-q_{n}}\left|\mathscr{F}_{x} g\right|^{2} d \xi \leqq C\|g\|_{z, s-\mu_{j}-q_{n} / 2, \gamma}^{2}
$$

and (see 3.5.8)

$$
I_{2}=\int H_{j}(z, \xi)\left|\mathscr{F}_{x} g\right|^{2} d \xi \leqq C \int h(z, \xi)^{2 s-2 \mu_{j}-q_{n}}\left|\mathscr{F}_{x} g\right|^{2} d \xi \leqq C\|g\|_{z, s-\mu_{j}-q_{n} / 2, \gamma}^{2} .
$$

It therefore follows from (1) that

and hence

$$
E K_{j} g \in H_{z}^{s}\left(\boldsymbol{R}^{n}\right)
$$

moreover,

$$
K_{j} g=R_{+} E K_{j} g \in H_{z}^{s}\left(R_{+}^{n}\right)
$$

Consequently,

$$
\left\|K_{j} g\right\|_{z, s,+} \leqq C\|g\|_{z, s-\mu_{j}-q_{n} / 2, \gamma}^{2}
$$

$$
K_{j} \in \mathscr{L}\left(H_{z}^{s-\mu_{j}-q_{n} / 2}\left(\boldsymbol{R}^{n-1}\right) ; H_{z}^{s}\left(\boldsymbol{R}_{+}^{n}\right)\right) .
$$

3.6.3. Let us now set

$$
K^{0} F=K_{0} f+\sum_{j=1}^{\varkappa} K_{j}\left(g_{j}-\gamma_{0} B_{j}^{0}(z, D) K_{0} f\right), \quad F=\left(f, g_{1}, \ldots, g_{\varkappa}\right) \in \mathscr{H}_{z}^{s}\left(\boldsymbol{R}_{+}^{n}, \boldsymbol{R}^{n-1}\right) .
$$

Then $K^{0}$ is an operator on $\mathscr{H}_{z}^{s}\left(\boldsymbol{R}_{+}^{n}, \boldsymbol{R}^{n-1}\right)$, valued in $H_{z}^{s}\left(\boldsymbol{R}_{+}^{n}\right)$, by virtue of 3.6.1 and 3.6.2, and furthermore

$$
\left\|K^{0} F\right\|_{z, s,+} \leqq C\|f\|_{z, s-\mu,+}+C \sum_{j=1}^{x}\left(\left\|g_{j}\right\|_{z, s-\mu_{j}-q_{n} / 2, \gamma}+\left\|\gamma_{0} B_{j}^{0}(z, D) K_{0} f\right\|_{z, s-\mu_{j}-q_{n} / 2, \gamma}\right),
$$

where (see Lemmas 1.9 and 3.2)

Thus we have

$$
\left\|\gamma_{0} B_{j}^{0}(z, D) K_{0} f\right\|_{z, s-\mu_{j}-q_{n} / 2, \gamma} \leqq C\|f\|_{z, s-\mu,+} .
$$

$$
K^{0} \in \mathscr{L}\left(\mathscr{H}_{z}^{s}\left(\boldsymbol{R}_{+}^{n}, \boldsymbol{R}^{n-1}\right) ; H_{z}^{s}\left(\boldsymbol{R}_{+}^{n}\right)\right) .
$$


3.6.4. The operator $K^{0}$ has the property

$$
P^{0}(z, D) K^{0} F=F, \quad F=\left(f, g_{1}, \ldots, g_{\varkappa}\right) \in \mathscr{H}_{z}^{s}\left(\boldsymbol{R}_{+}^{n}, \boldsymbol{R}^{n-1}\right) .
$$

To see this, let $F=\left(f, g_{1}, \ldots, g_{\varkappa}\right) \in \mathscr{H}_{z}^{s}\left(\boldsymbol{R}_{+}^{n}, \boldsymbol{R}^{n-1}\right)$ and set $u=K^{0} F \in H_{z}^{s}\left(\boldsymbol{R}_{+}^{n}\right)$. Then we have

$$
A^{0}(z, D) u=A^{0}(z, D) K_{0} f+\sum_{j=1}^{\varkappa} A^{0}(z, D) K_{j}\left(g_{j}-\gamma_{0} B_{j}^{0}(z, D) K_{0} f\right),
$$

where (note that $A^{0}(z, D)$ and $R_{+}$commute)

$$
\begin{aligned}
A^{0}(z, D) K_{0} f & =A^{0}(z, D) R_{+} \mathscr{F}_{\eta} A^{0}(z, \eta)^{-1} \mathscr{F} E f \\
& =R_{+} \mathscr{F}_{\eta} A^{0}(z, \eta) A^{0}(z, \eta)^{-1} \mathscr{F} E f \\
& =f
\end{aligned}
$$

and (see 3.3.7)

$$
\begin{aligned}
A^{0}(z, D) K_{j}\left(g_{j}-\gamma_{0} B_{j}^{0}(z, D) K_{0} f\right) & =A^{0}(z, D) \mathscr{F}_{\xi}\left(N_{j} \mathscr{F}_{x}\left(g_{j}-\gamma_{0} B_{j}^{0}(z, D) K_{0} f\right)\right) \\
& =\mathscr{F}_{\xi}\left(\left(A^{0}\left(z, \xi, D_{t}\right) N_{j}\right) \mathscr{F}_{x}\left(g_{j}-\gamma_{0} B_{j}^{0}(z, D) K_{0} f\right)\right) \\
& =0,
\end{aligned}
$$

so that

$$
A^{0}(z, D) u=f
$$

By (12) of 3.3, we obtain further

whence

$$
\begin{aligned}
& \gamma_{0} B_{k}^{0}(z, D) K_{j}\left(g_{j}-\gamma_{0} B_{j}^{0}(z, D) K_{0} f\right) \\
& =\mathscr{F}_{\xi}\left(\left(\gamma_{0} B_{k}^{0}\left(z, \xi, D_{t}\right) N_{j}\right) \mathscr{F}_{x}\left(g_{j}-\gamma_{0} B_{j}^{0}(z, D) K_{0} f\right)\right) \\
& =\delta_{j k}\left(g_{j}-\gamma_{0} B_{j}^{0}(z, D) K_{0} f\right),
\end{aligned}
$$

as required.

$$
\begin{aligned}
\gamma_{0} B_{k}^{0}(z, D) u & =\gamma_{0} B_{k}^{0}(z, D) K_{0} f+\sum_{j=1}^{\varkappa} \delta_{j k}\left(g_{j}-\gamma_{0} B_{j}^{0}(z, D) K_{0} f\right) \\
& =g_{k}
\end{aligned}
$$

It thus follows that the operator $P^{0}(z, D)$ is a continuous bijective linear map and therefore an isomorphism from $H_{z}^{s}\left(\boldsymbol{R}_{+}^{n}\right)$ onto $\mathscr{H}_{z}^{s}\left(\boldsymbol{R}_{+}^{n}, \boldsymbol{R}^{n-1}\right)$, with inverse $K^{0}$. The continuity of $K^{0}$ was, in fact, found also directly in 3.6.3.

\section{The general case}

In this section we shall generalize the results of Theorem 3.4 to cover the case of nonhomogeneous operators.

4.1. We shall need the following two lemmas. 
4.1.1. Lemma. Suppose $s_{1}>s_{2}>s_{3} \geqq 0$. Given any $\varepsilon>0$ there is a constant $C(\varepsilon)>0$ such that

for all $z \in \boldsymbol{C}$.

$$
\|u\|_{z, s_{2},+} \leqq \varepsilon\|u\|_{z, s_{1},+}+C(\varepsilon)\|u\|_{z, s_{3},+}, \quad u \in H^{s_{1}}\left(\boldsymbol{R}_{+}^{n}\right),
$$

Proof. From the well-known inequality (equal to the above with $z=0$ )

we obtain

$$
\|u\|_{s_{2},+} \leqq \varepsilon\|u\|_{s_{1},+}+C(\varepsilon)\|u\|_{s_{3},+}, \quad u \in H^{s_{1}}\left(R_{+}^{n}\right),
$$

$$
\|u\|_{z, \mathrm{~s} 2,+} \sim\|u\|_{s_{2},+}+\langle z\rangle^{s_{2}}\|u\|_{+} \leqq \varepsilon\|u\|_{s_{1},+}+C(\varepsilon)\|u\|_{s_{3},+}+\langle z\rangle^{s_{2}}\|u\|_{+} .
$$

The assertion follows therefore from the inequality

$$
\langle z\rangle^{s_{2}} \leqq \varepsilon\langle z\rangle^{s_{1}}+C(\varepsilon)\langle z\rangle^{s_{3}},
$$

which in turn is a consequence of the elementary inequality

$$
a b \leqq \frac{1}{p} a^{p}+\frac{1}{p^{\prime}} b^{p^{\prime}}, \quad a, b>0, \quad p>1, \quad p^{\prime}=p /(p-1) ;
$$

as a matter of fact, choose

$$
p=\frac{s_{1}-s_{3}}{s_{2}-s_{3}}, \quad a=(p \varepsilon)^{1 / p}\langle z\rangle^{s_{1} / p}, \quad b=(p \varepsilon)^{-1 / p}\langle z\rangle^{s_{3} / p^{\prime}} .
$$

4.1.2. Lemma. Let $s \geqq 0$ and $r>0$. To every $\varepsilon>0$ there is $C(\varepsilon)>0$ such that

$$
\sum_{\langle\alpha, q\rangle<r}\left\|D^{\alpha} u\right\|_{z, s,+} \leqq \varepsilon\|u\|_{z, s+r,+}+C(\varepsilon)\|u\|_{+}, \quad u \in H^{s+r}\left(R_{+}^{n}\right),
$$

for all $z \in \boldsymbol{C}$.

Proof. By Lemma 1.10 we have

If we now put

$$
\left\|D^{\alpha} u\right\|_{z, s,+} \leqq C\|u\|_{z, s+\langle\alpha, q\rangle,+} .
$$

$$
k=\max \left\{\langle\alpha, q\rangle \mid \alpha \in N^{n},\langle\alpha, q\rangle<r\right\}<r .
$$

it follows from Lemma 1.5 that

$$
\sum_{\langle\alpha, q\rangle<r}\|u\|_{z, s+\langle\alpha, q\rangle,+} \leqq C\|u\|_{z, s+k,+},
$$

where, by virtue of Lemma 4.1.1,

$$
\|u\|_{z, s+k,+} \leqq \varepsilon\|u\|_{z, s+r,+}+C(\varepsilon)\|u\|_{+} .
$$

4.2. In the general case we have to consider operators of the form

$$
P(z, D)=P^{0}(z, D)+P^{00}(z, D),
$$

where $P^{0}(z, D)$, the principal part of $P(z, D)$, is defined as in 3.1 , and $P^{00}(z, D)$ 
is given by

$$
P^{00}(z, D)=\left(A^{00}(z, D), \gamma_{0} B^{00}(z, D)\right)=\left(A^{00}(z, D), \gamma_{0} B_{1}^{00}(z, D), \ldots, \gamma_{0} B_{\varkappa}^{00}(z, D)\right)
$$

with

$$
\begin{aligned}
& A^{00}(z, D)=\sum_{k q_{0}+\langle\alpha, q\rangle<\mu} a_{k \alpha} z^{k} D^{\alpha}, \\
& B_{j}^{00}(z, D)=\sum_{k q_{0}+\langle\alpha, q\rangle<\mu_{j}} b_{j k \alpha} z^{k} D^{\alpha} ;
\end{aligned}
$$

here the coefficients $a_{k \alpha}$ and $b_{j k \alpha}$ are complex constants, of course.

4.3. Theorem. Suppose that $P^{0}(z, D)$ satisfies Conditions $(Q E D)$ and $(C C)$, and let $s \in S$. Then there is $\varrho>0$ such that, for some constant $C>0$,

$$
\|u\|_{z, s,+} \leqq C\|P(z, D) u\|_{z, s}, \quad u \in H^{s}\left(\boldsymbol{R}_{+}^{n}\right),
$$

for all $z \in Z,\langle z\rangle \geqq \varrho$.

Proof. In what follows let $u$ be any function in $H^{s}\left(\boldsymbol{R}_{+}^{n}\right)$ and let $z \in Z \backslash\{0\}$.

4.3.1. According to Theorem 3.4 we first have

which yields

$$
\|u\|_{z, s,+} \leqq C\left\|P^{0}(z, D) u\right\|_{z, s},
$$

(1)

$$
\begin{aligned}
\|u\|_{z, s,+} \leqq C_{1}\left(\|P(z, D) u\|_{z, s}\right. & +\left\|A^{00}(z, D) u\right\|_{z, s-\mu,+} \\
& \left.+\sum_{j=1}^{\varkappa}\left\|\gamma_{0} B_{j}^{00}(z, D) u\right\|_{z, s-\mu_{j}-q_{n} / 2, \gamma}\right) .
\end{aligned}
$$

4.3.2. Given any $\varepsilon>0$ we obtain, by virtue of Lemma 4.1.2,

$$
\begin{gathered}
\left\|A^{00}(z, D) u\right\|_{z, s-\mu,+} \leqq C_{2} \sum_{k<m_{0}}|z|^{k} \sum_{\langle\alpha, q\rangle<\mu-k q_{0}}\left\|D^{\alpha} u\right\|_{z, s-\mu,+} \\
\leqq C_{2} \varepsilon \sum_{k<m_{0}}\langle z\rangle^{k q_{0}}\|u\|_{z, s-k q_{0},+}+C_{3}(\varepsilon) \sum_{k<m_{0}}\langle z\rangle^{k q_{0}}\|u\|_{+} \cdot
\end{gathered}
$$

Since

$$
\sum_{k<m_{0}}\langle z\rangle^{k q_{0}}\|u\|_{+} \leqq C\left(1+\langle z\rangle^{\mu-q_{0}}\right)\langle z\rangle^{-s}\|u\|_{z, s,+},
$$

we have, by Lemma 1.7,

$$
\left\|A^{00}(z, D) u\right\|_{z, s-\mu,+} \leqq\left(C_{4} \varepsilon+C_{5}(\varepsilon)\left(1+\langle z\rangle^{\mu-q_{0}}\right)\langle z\rangle^{-s}\right)\|u\|_{z, s,+} .
$$

Fix now $\varepsilon>0$ satisfying

$$
C_{4} \varepsilon \leqq \frac{1}{8 C_{1}}
$$

and choose $\varrho_{0}>0$ such that

$$
C_{5}(\varepsilon) \frac{1+\varrho_{0}^{\mu-q_{0}}}{\varrho_{0}^{s}} \leqq \frac{1}{8 C_{1}}
$$


Then it follows from (2) that

$$
\left\|A^{00}(z, D) u\right\|_{z, s-\mu,+} \leqq \frac{1}{4 C_{1}}\|u\|_{z, s,+}
$$

for all $z \in Z,\langle z\rangle \geqq \varrho_{0}$.

4.3.3. Next, in view of Lemma 1.9, we find that

$$
\left\|\gamma_{0} B_{j}^{00}(z, D) u\right\|_{z, s-\mu_{j}-q_{n} / 2, \gamma} \leqq C_{6} \sum_{k q_{0}<\mu_{j}}|z|^{k} \sum_{\langle\alpha, q\rangle<\mu_{j}-k q_{0}}\left\|D^{\alpha} u\right\|_{z, s-\mu_{j},+} .
$$

Hence, given any $\varepsilon>0$, we obtain again from Lemma 4.1.2

Since

$$
\begin{aligned}
& \left\|\gamma_{0} B_{j}^{00}(z, D) u\right\|_{z, s-\mu_{j}-q_{n} / 2, \gamma} \\
& \leqq C_{6} \varepsilon \sum_{k q_{0}<\mu_{j}}\langle z\rangle^{k q_{0}}\|u\|_{z, s-k q_{0},+}+C_{7}(\varepsilon) \sum_{k q_{0}<\mu_{j}}\langle z\rangle^{k q_{0}}\|u\|_{+} \cdot
\end{aligned}
$$

$$
\sum_{k q_{0}<\mu_{j}}\langle z\rangle^{k q_{0}}\|u\|_{+} \leqq C\left(1+\langle z\rangle^{\mu_{j}}\right)\langle z\rangle^{-s}\|u\|_{z, s,+}
$$

(obviously, this inequation could be strengthened), we have, as above,

$$
\left\|\gamma_{0} B_{j}^{00}(z, D) u\right\|_{z, s-\mu_{j}-q_{n} / 2, \gamma} \leqq\left(C_{8} \varepsilon+C_{9}(\varepsilon)\left(1+\langle z\rangle^{\mu_{j}}\right)\langle z\rangle^{-s}\right)\|u\|_{z, s,+} .
$$

If we now take $\varepsilon>0$ so small that

and choose $\varrho_{j}>0$ such that

$$
C_{8} \varepsilon \leqq \frac{1}{8 x C_{1}}
$$

then it follows from (4) that

$$
C_{9}(\varepsilon) \frac{1+\varrho_{j}^{\mu_{j}}}{\varrho_{j}^{s}} \leqq \frac{1}{8 \varkappa C_{1}},
$$

for all $z \in Z,\langle z\rangle \geqq \varrho_{j}$.

$$
\left\|\gamma_{0} B_{j}^{00}(z, D) u\right\|_{z, s-\mu_{j}-q_{n} / 2, \gamma} \leqq \frac{1}{4 \varkappa C_{1}}\|u\|_{z, s,+}
$$

4.3.4. To complete the proof, it is now enough to define $\varrho=\max \left\{\varrho_{0}, \varrho_{1}, \ldots, \varrho_{\varkappa}\right\}$. Indeed, combining (1), (3), and (5), we have

and thus

$$
\|u\|_{z, s,+} \leqq C_{1}\|P(z, D) u\|_{z, s}+\frac{1}{4}\|u\|_{z, s,+}+\sum_{j=1}^{\varkappa} \frac{1}{4 \varkappa}\|u\|_{z, s,+}
$$

for all $z \in Z,\langle z\rangle \geqq \varrho$.

$$
\|u\|_{z, s,+} \leqq C\|\mid P(z, D) u\|_{z, s}
$$

4.4. Theorem. If $P^{0}(z, D)$ satisfies Conditions (QED) and (CC), and if $s \in S$, then there is $\varrho>0$ such that, for every $z \in Z$ with $\langle z\rangle \geqq \varrho$, the operator $P(z, D)$ is an isomorphism of $H_{z}^{s}\left(\boldsymbol{R}_{+}^{n}\right)$ onto $\mathscr{H}_{z}^{s}\left(\boldsymbol{R}_{+}^{n}, \boldsymbol{R}^{n-1}\right)$.

Proof. We shall show that $P(z, D)$ can be represented as the product of $P^{0}(z, D)$ and a $\mathscr{H}_{z}^{s}\left(\boldsymbol{R}_{+}^{n}, \boldsymbol{R}^{n-1}\right)$-automorphism. 
4.4.1. Let $z \in Z \backslash\{0\}$. According to Theorem 3.4 the operator $P^{0}(z, D)$ is an isomorphism of $H_{z}^{s}\left(\boldsymbol{R}_{+}^{n}\right)$ onto $\mathscr{H}_{z}^{s}\left(\boldsymbol{R}_{+}^{n}, \boldsymbol{R}^{n-1}\right)$. As in 3.6 , let $K^{0}$ denote its inverse. Then we can write

$$
P(z, D)=P^{0}(z, D)+P^{00}(z, D)=\left(I_{s}+P^{00}(z, D) K^{0}\right) P^{0}(z, D),
$$

where $I_{s}$ is the identity mapping on $\mathscr{H}_{z}^{s}\left(\boldsymbol{R}_{+}^{n}, \boldsymbol{R}^{n-1}\right)$.

4.4.2. Let $u \in H_{z}^{s}\left(\boldsymbol{R}_{+}^{n}\right)$. Given any $\varepsilon>0$ we have, by (2) of 4.3,

$$
\left\|A^{00}(z, D) u\right\|_{z, s-\mu,+} \leqq\left(C_{1} \varepsilon+C_{2}(\varepsilon)\left(1+\langle z\rangle^{\mu-q_{0}}\right)\langle z\rangle^{-s}\right)\|u\|_{z, s,+}
$$

and, by (4) of 4.3,

$$
\left\|\gamma_{0} B_{j}^{00}(z, D) u\right\|_{z, s-\mu_{j}-q_{n} / 2, \gamma} \leqq\left(C_{3} \varepsilon+C_{4}(\varepsilon)\left(1+\langle z\rangle^{\mu}\right)\langle z\rangle^{-s}\right)\|u\|_{z, s,+} .
$$

Fix $\sigma, 0<\sigma<1$. Next, choose $\varepsilon>0$ satisfying

$$
C_{1} \varepsilon \leqq \frac{\sigma}{2 \sqrt{2}\left\|K^{0}\right\|}, \quad C_{3} \varepsilon \leqq \frac{\sigma}{2 \sqrt{2 \varkappa}\left\|K^{0}\right\|}
$$

$\left(\left\|K^{0}\right\|\right.$ is of course the norm of $\left.K^{0} \in \mathscr{L}\left(\mathscr{H}_{z}^{s}\left(\boldsymbol{R}_{+}^{n}, \boldsymbol{R}^{n-1}\right) ; H_{z}^{s}\left(\boldsymbol{R}_{+}^{n}\right)\right)\right)$, and then $\varrho_{0}$ such that

$$
C_{2}(\varepsilon) \frac{1+\varrho_{0}^{\mu-q_{0}}}{\varrho_{0}^{s}} \leqq \frac{\sigma}{2 \sqrt{2}\left\|K^{0}\right\|}
$$

and $\varrho_{j}$ such that

$$
C_{4}(\varepsilon) \frac{1+\varrho_{j}^{\mu_{j}}}{\varrho_{j}^{s}} \leqq \frac{\sigma}{2 \sqrt{2 \varkappa}\left\|K^{0}\right\|} .
$$

Setting $\varrho=\max \left\{\varrho_{0}, \varrho_{1}, \ldots, \varrho_{x}\right\}$, we thus obtain

$$
\begin{aligned}
\left\|P^{00}(z, D) u\right\|_{z, s} & =\left(\left\|A^{00}(z, D) u\right\|_{z, s-\mu,+}^{2}+\sum_{j=1}^{\varkappa}\left\|\gamma_{0} B_{j}^{00}(z, D) u\right\|_{z, s-\mu_{j}-q_{,} / 2, \gamma}^{2}\right)^{1 / 2} \\
& \leqq \frac{\sigma}{\left\|K^{0}\right\|}\|u\|_{z, s,+}
\end{aligned}
$$

whenever $z \in Z$ with $\langle z\rangle \geqq \varrho$.

4.4.3. It now follows from (1) that

$$
\|\| P^{00}(z, D) K^{0} F\left\|_{z, s} \leqq \sigma\right\| \mid F\|\|_{z, s}, \quad F \in \mathscr{H}_{z}^{s}\left(\boldsymbol{R}_{+}^{n}, \boldsymbol{R}^{n-1}\right) .
$$

This implies that the inverse $\left(I_{s}+P^{00}(z, D) K^{0}\right)^{-1}$ exists and is continuous, and so is the operator

$$
K=K^{0}\left(I_{s}+P^{00}(z, D) K^{0}\right)^{-1} .
$$

Hence we reach the conclusion that, for every $z \in Z$ with $\langle z\rangle \geqq \varrho$, the operator

$$
P(z, D)=\left(I_{s}+P^{00}(z, D) K^{0}\right) P^{0}(z, D)
$$

is an isomorphism of $H_{z}^{s}\left(\boldsymbol{R}_{+}^{n}\right)$ onto $\mathscr{H}_{z}^{s}\left(\boldsymbol{R}_{+}^{n}, \boldsymbol{R}^{n-1}\right)$, with inverse $K$. 


\subsection{Two remarks}

4.5.1. As one can easily verify, Theorem 4.4 implies a known result (see Teorema 6.3 of V. del Prete-D. Fortunato [6]; cf. also Teorema 9.2 of S. Matarasso [5]) for the unique solvability in $H^{s}\left(\boldsymbol{R}_{+}^{n}\right)$ of the problem

$$
\left(A(D) u+\lambda u, \gamma_{0} B(D) u\right)=(f, g)
$$

with any $(f, g) \in \mathscr{H}^{s}\left(\boldsymbol{R}_{+}^{n}, \boldsymbol{R}^{n-1}\right)$ for every $\lambda \in \boldsymbol{C}$ with $\arg \lambda=$ constant, provided that $|\lambda|$ is sufficiently large. This shows that the assumptions usually made (see [6]) can mildly be reduced.

4.5.2. We conclude by mentioning one direction of generalizations: It is possible to consider also boundary problems for operators with symbols from more general classes of "parametrically" hypoelliptic polynomials, and for spaces with more general weight functions. In this connection note, for example, the problems considered by S. Matarasso [5] in the nonparametric case.

\section{References}

[1] Agmon, S., A. Douglis, and L. Nirenberg: Estimates near the boundary for solutions of elliptic partial differential equations satisfying general boundary conditions. I. - Comm. Pure Appl. Math. 12, 1959, 623-727.

[2] Agranovič, M. S., and M. I. Višik : Elliptic problems with a parameter and parabolic problems of general type. - Russian Math. Surveys 19, 1964, no. 3, 53-157.

[3] Hörmander, L.: Linear partial differential operators. - [Fourth printing.] Die Grundlehren der mathematischen Wissenschaften 116. Springer-Verlag, Berlin-Heidelberg-New York, 1976.

[4] Lions, J. L., and E. Magenes: Non-homogeneous boundary value problems and applications I. - Die Grundlehren der mathematischen Wissenschaften 181. Springer-Verlag, Berlin-Heidelberg-New York, 1972.

[5] Matarasso, S.: Esistenza ed unicità delle soluzioni di una classe di problemi ipoellittici. - Ann. Mat. Pura Appl. (4) 109, 1976, 39-87.

[6] Del Prete, V., and D. Fortunato: Teoremi di regolarizzazione per problemi quasi ellittici in un semispazio ed applicazioni. - Ricerche Mat. 23, 1974, 87-128.

[7] SLobodeckir, L. N.: Generalized Sobolev spaces and their application to boundary problems for partial differential equations. - Amer. Math. Soc. Transl. (2) 57, 1966, 207-275.

[8] Troisi, M.: Problemi al contorno con condizioni omogenee per le equazioni quasi-ellittiche. - Ann. Mat. Pura Appl. (4) 90, 1971, 331-412.

[9] Volevič, L. R., and B. P. PaneJah: Certain spaces of generalized functions and embedding theorems. - Russian Math. Surveys 20, 1965, no. 1, 1-73.

University of Jyväskylä

Department of Mathematics

Seminaarinkatu 15

SF-40100 Jyväskylä 10

Finland

Received 10 December 1979 